JOURNAL OF INTEGRAL EQUATIONS

AND APPLICATIONS

Volume 21, Number 4, Winter 2009

\title{
PROJECTION METHODS FOR FREDHOLM INTEGRAL EQUATIONS ON THE REAL SEMIAXIS
}

\author{
C. LAURITA AND G. MASTROIANNI \\ Communicated by Giovanni Monegato
}

\begin{abstract}
Numerical procedures to solve Fredholm integral equations of the second kind on the real semiaxis are proposed. Their stability and convergence are proved and error estimates in $L^{p}$ weighted norm are given. Numerical examples are also included.
\end{abstract}

1. Introduction. Let us consider Fredholm integral equations of the second kind on unbounded intervals of the following type

$$
f(y)-\int_{0}^{\infty} k(x, y) f(x) w(x) d x=g(y),
$$

where $w(x)=x^{\alpha} e^{-x^{\beta}}, \alpha>-1, \beta>1 / 2, k(x, y)$ and $g(x)$ are known functions and $f(x)$ is an unknown function. We want to study equation (1.1) in the spaces $L_{u}^{p}, 1<p<\infty$ and $u(x)=x^{\gamma} e^{-x^{\beta} / 2}, \gamma>-1 / p$.

When $y \in(-1,1), w$ is a Jacobi weight and the integral is defined on the bounded interval $(-1,1)$, there is a large literature about the numerical solution of such kind of equations (see, for example $[\mathbf{1}, \mathbf{3}$, $\mathbf{6 , 1 7}])$. In [5] Fredholm integral equations of the second kind on the interval $(0,+\infty)$ are considered. Here the weight $w(x)$ is the classical Laguerre weight $(\beta=1)$ and the space is $L_{\sqrt{w}}^{2}$.

In this paper both $w(x)$ and $u(x)$ are more general weights. Moreover the index $p$ can assume any real value in $(1,+\infty)$. The main difficulties

\footnotetext{
2000 AMS Mathematics subject classification. Primary 65R20, 45E05, 41A05, 41A10.

Keywords and phrases. Fredholm integral equations, projection method, Nyström method, Lagrange interpolation, condition number.

Received by the editors on April 30, 1007, and in revised form on September 14, 2007.

DOI:10.1216/JIE-2009-21-4-559 Copyright (C2009 Rocky Mountain Mathematics Consortium
} 
by going from the $L_{\sqrt{w}}^{2}$ case in [5] to the $L_{u}^{p}$ case is to have and to apply new suitable tools of approximation theory (estimates of Lagrange interpolation error, polynomial inequalities, etc.) which hold in such more general spaces of function.

The aim is to introduce a new numerical procedure to approximate the solution of (1.1), if it is unisolvent. This procedure is based on the theory of the polynomial approximation in $L_{u}^{p}$ and leads to the resolution of a system of linear equations.

Such kind of approach involves some difficulties. In the first place, in $L_{u}^{p}(0,+\infty)$ there is no uniformly bounded sequence of projections $P_{m}: L_{u}^{p}(0,+\infty) \longrightarrow \mathbb{P}_{m}$, being $\mathbb{P}_{m}$ the subspace of all algebraic polynomials of degree at most $m$, for any value of $p$.

Furthermore, when $w(x)=x^{\alpha} e^{-x^{\beta}}$, with $\beta \neq 1$, i.e. $w(x)$ is not the classical generalized Laguerre weight, the nodes and weights of the quadrature formula cannot be computed by using a standard procedure because, in this case, the coefficients of the recurrence formula satisfied by the system of orthonormal polynomials $\left\{p_{m}(w)\right\}_{m}$ are not known.

In this paper, to overcome the first problem, we consider special sequences of Lagrange interpolation operators, based on a part of the zeros of the polynomial $p_{m}(w)$ (see Section 2.2). Moreover we use (truncated) quadrature rules to approximate the integral

$$
\int_{0}^{\infty} k(x, y) f(x) w(x) d x
$$

when $k(x, y)$ is sufficiently smooth. This idea, introduced in [10] has been used in different contexts $[\mathbf{5}, \mathbf{1 2}]$.

The numerical computation of nodes and coefficients of these Gaussian type quadrature formulas, related to generalized Laguerre weight $w(x)$, is carried out by using a Mathematica package appearing in [2].

We prove that the linear system related to the discrete operator is well conditioned. The approximating solution converges to the exact one and error estimates are given. As a consequence we, also, prove that the Nyström method is stable and convergent.

The paper is organized as follows. In Section 2 we introduce some notations and obtain some preliminary results. In Section 3 we describe our numerical methods. In Section 4 we present some numerical tests while Section 5 contains the proofs of the main results. 


\section{Notations and preliminary results.}

2.1. Spaces of functions. For $1<p<+\infty, S \subseteq(0,+\infty)$ let $L^{p}(S)$ be defined in the usual way and, with $u(x)=x^{\gamma} e^{-x^{\beta} / 2}, \gamma>-1 / p$, $\beta>1 / 2$, let $L_{u}^{p}(S)$ be the collection of all measurable functions $f$ such that $f u \in L^{p}(S)$. The norm in $L_{u}^{p}(S)$ is defined by

$$
\|f\|_{L_{u}^{p}(S)}=\left(\int_{S}|f u|^{p}(x) d x\right)^{1 / p} .
$$

For simplicity of notations, we set $L_{u}^{p}=L_{u}^{p}((0,+\infty))$.

When $p=+\infty$, we define the space

$$
L_{u}^{\infty}:=C_{u}=\left\{f \in C^{0}((0,+\infty)): \lim _{\substack{x \rightarrow 0 \\ x \rightarrow \infty}}(f u)(x)=0\right\}
$$

both equipped with the norm

$$
\|f\|_{L_{u}^{\infty}}=\|f\|_{C_{u}}=\sup _{x \geq 0}|(f u)(x)| .
$$

Moreover, let $W_{r}^{p}(u)$ be the weighted Sobolev-type space of the order $r \in \mathbb{N}, r \geq 1$, defined by

$$
W_{r}^{p}(u)=\left\{f \in L_{u}^{p}:\left\|f^{(r)} \varphi^{r} u\right\|_{p}<+\infty\right\}, \quad \varphi(x)=\sqrt{x},
$$

and equipped with the norm

$$
\|f\|_{W_{r}^{p}(u)}=\|f u\|_{p}+\left\|f^{(r)} \varphi^{r} u\right\|_{p} .
$$

Let us, also, consider Zygmund-type spaces defined as follows

$$
Z_{s}^{p}(u)=\left\{f \in L_{u}^{p}([0,+\infty)):\|f\|_{Z_{s}^{p}(u)}<\infty\right\}
$$

where

$$
\|f\|_{Z_{s}^{p}(u)}=\|f u\|_{p}+\sup _{t>0} \frac{\Omega_{\varphi}^{r}(f, t)_{u, p}}{t^{s}}, \quad r>s,
$$

with the main part of modulus of continuity

$$
\Omega_{\varphi}^{r}(f, t)_{u, p}=\sup _{0<h \leq t}\left\|\left(\vec{\Delta}_{h \varphi}^{r} f\right) u\right\|_{L^{p}\left(I_{r h}\right)},
$$


$\varphi(x)=\sqrt{x}, I_{r h}=\left[8(r h)^{2}, \mathcal{C} h^{*}\right], \mathcal{C}$ arbitrary fixed constant, $h^{*}=$ $1 /\left(h^{2 /(2 \beta-1)}\right)$ and

$$
\vec{\Delta}_{h \varphi}^{r} f(x)=\sum_{i=0}^{r}(-1)^{i}\left(\begin{array}{l}
r \\
i
\end{array}\right) f\left(x+\left(\frac{r}{2}-i\right) h \sqrt{x}\right) .
$$

Denote by $\mathbb{P}_{m}$ the set of all algebraic polynomials of degree at most $m$ and by $E_{m}(f)_{u, p}=\inf _{P \in \mathbb{P}_{m}}\|(f-P) u\|_{p}$ the error of best approximation by algebraic polynomials of degree at most $m$. In [14] the authors proved the following estimate, holding for functions $f \in W_{r}^{p}(u)$,

$$
E_{m}(f)_{u, p} \leq \mathcal{C}\left(\frac{\sqrt{a_{m}}}{m}\right)^{r}\left\|f^{(r)} \varphi^{r} u\right\|_{p}, \quad \mathcal{C} \neq \mathcal{C}(m, f)
$$

where $a_{m}$ is the Mhaskar-Rachmanov-Saff number $a_{m}=a_{m}(u)=$ $\mathcal{C}(\alpha, \beta) m^{1 / \beta}$ and the constant $\mathcal{C}(\alpha, \beta)$ (see $\left.[13,16]\right)$ is not essential for our aims.

Here and in the sequel we denote by $\mathcal{C}$ a positive constant which may assume different values in different formulae. We write $\mathcal{C} \neq \mathcal{C}(a, b, \ldots)$ if $\mathcal{C}$ is independent of the parameters $a, b, \ldots$ If $A, B \geq 0$ are quantities depending on some parameters, we write $A \sim B$, if there exists a positive constant $\mathcal{C}$ independent of the parameters $A$ and $B$ such that $(B / \mathcal{C}) \leq A \leq \mathcal{C} B$

2.2. Lagrange interpolation and Fourier sums. Let $w(x)=$ $x^{\alpha} e^{-x^{\beta}}, x>0, \alpha>-1, \beta>1 / 2$ be a generalized Laguerre weight, $u(x)=x^{\gamma} e^{-x^{\beta} / 2}, \gamma>-1 / p$.

Let $\left\{p_{m}(w)\right\}_{m}$ be the sequence of orthonormal polynomials with respect to the weight $w(x)$ having positive leading coefficient, and let $x_{1}, x_{2}, \ldots, x_{m},\left(x_{k}=x_{m k}\right)$ be the zeros of $p_{m}(w)$. We recall that $\mathcal{C}\left(a_{m} / m^{2}\right) \leq x_{1}<x_{2}<\cdots<x_{m}<a_{m}$, with $a_{m}=a_{m}(w) \sim m^{1 / \beta}$ the M-R-S number with respect to the weight $w(x)$ (see [14]), and for $\theta \in(0,1)$ fixed, we define the integer $j=j(m)$ by

$$
x_{j}=\min _{1 \leq k \leq m}\left\{x_{k}: x_{k} \geq \theta a_{m}\right\}
$$

with $m$ sufficiently large (say $m>m_{0}$ ). Then we define the function $f_{j}(x)=f(x) \Phi_{j}(x)$ with $\Phi_{j}$ the characteristic function of the interval 
$\left[0, x_{j}\right]$. For any continuous function $f$ on $[0,+\infty)$, i.e., $f \in C\left(\mathbb{R}^{+}\right)$, by definitions, $f_{j}=f$ in $\left[0, x_{j}\right]$ and $f_{j}=0$ in $\left(x_{j},+\infty\right)$. Let us introduce a Lagrange polynomial $L_{m+1}^{*}(w, f)$ which interpolates $f \in C\left(\mathbb{R}^{+}\right)$on the $m+1$ points $x_{1}, x_{2}, \ldots, x_{m}, a_{m}$, i.e.

$$
L_{m+1}^{*}(w, f ; x)=\sum_{k=1}^{m} l_{k}(x) \frac{a_{m}-x}{a_{m}-x_{k}} f\left(x_{k}\right)+\frac{p_{m}(w, x)}{p_{m}\left(w, a_{m}\right)} f\left(a_{m}\right)
$$

where $l_{k}(x)$ are the fundamental Lagrange polynomials based on the zeros of $p_{m}(w)$. One can also represent $L_{m+1}^{*}(w, f)$ as

$$
L_{m+1}^{*}(w, f ; x)=\sum_{k=1}^{m+1} \tilde{l}_{k}(x) f\left(x_{k}\right)
$$

with

$$
\tilde{l}_{k}(x)=l_{k}(x) \frac{a_{m}-x}{a_{m}-x_{k}}, \quad k=1, \ldots, m,
$$

and

$$
\tilde{l}_{m+1}(x)=\frac{p_{m}(w, x)}{p_{m}\left(w, a_{m}\right)}
$$

the fundamental Lagrange polynomials on the nodes $x_{1}, x_{2}, \ldots, x_{m}, a_{m}$ and $x_{m+1}=a_{m}$.

Moreover, for our aims, we will consider the polynomial interpolating the truncated function $f_{j}$, i.e.,

$$
L_{m+1}^{*}\left(w, f_{j} ; x\right)=\sum_{k=1}^{j} l_{k}(x) \frac{a_{m}-x}{a_{m}-x_{k}} f\left(x_{k}\right)
$$

or

$$
L_{m+1}^{*}\left(w, f_{j} ; x\right)=\sum_{k=1}^{j} \tilde{l}_{k}(x) f\left(x_{k}\right)
$$

and use the following estimates it satisfies (see [7]). 
Lemma 2.1. Let $1<p<+\infty$ and $v^{\sigma}(x)=x^{\sigma}$. If the weights $w(x)=v^{\alpha}(x) e^{-x^{\beta}}$ and $u(x)=v^{\gamma}(x) e^{-x^{\beta} / 2}$ satisfy the conditions

$$
\frac{\alpha}{2}+\frac{1}{4}-\frac{1}{p}<\gamma<\frac{\alpha}{2}+\frac{5}{4}-\frac{1}{p}
$$

then, for all functions $f \in C\left(\mathbb{R}^{+}\right)$, we have

$$
\left\|L_{m+1}^{*}\left(w, f_{j}\right) u\right\|_{p} \leq \mathcal{C}\left(\sum_{k=1}^{j} \Delta x_{k}|f u|^{p}\left(x_{k}\right)\right)^{1 / p},
$$

where $\Delta x_{k}=x_{k+1}-x_{k}$ and $\mathcal{C} \neq \mathcal{C}(m, f)$.

Let us remark that conditions (2.5) are equivalent to the following ones

$$
\begin{aligned}
\frac{v^{\gamma}}{\sqrt{v^{\alpha} \varphi}} & \in L^{p}(0,1) \quad \text { and } \quad \frac{\sqrt{v^{\alpha} \varphi}}{v^{\gamma}} \in L^{q}(0,1), \\
\varphi(x) & =\sqrt{x}, \quad q=\frac{p}{p-1} .
\end{aligned}
$$

Lemma 2.2. For any function $f \in C\left(\mathbb{R}^{+}\right), 1<p<+\infty$, we have

$$
\begin{aligned}
& \left(\sum_{k=1}^{j} \Delta x_{k}|f u|^{p}\left(x_{k}\right)\right)^{1 / p} \\
& \quad \leq \mathcal{C}\left[\|f u\|_{L^{p}\left(0, x_{j+1}\right)}+\left(\frac{\sqrt{a_{m}}}{m}\right)^{1 / p} \int_{0}^{\sqrt{a_{m}} / m} \frac{\Omega_{\varphi}^{r}(f, t)_{u, p}}{t^{1+(1 / p)}} d t\right]
\end{aligned}
$$

where $\Delta x_{k}=x_{k+1}-x_{k}$ and $\mathcal{C} \neq \mathcal{C}(m, f)$.

Lemma 2.3. Let $1<p<+\infty$. Under assumptions (2.5), for any $f \in Z_{s}^{p}(u), s>1 / p$, we have

$$
\left\|\left[f-L_{m+1}^{*}\left(w, f_{j}\right)\right] u\right\|_{p} \leq \mathcal{C}\left(\frac{\sqrt{a_{m}}}{m}\right)^{s}\|f\|_{Z_{s}^{p}(u)}
$$

and for any $f \in W_{r}^{p}(u)$

$$
\left\|\left[f-L_{m+1}^{*}\left(w, f_{j}\right)\right] u\right\|_{p} \leq \mathcal{C}\left(\frac{\sqrt{a_{m}}}{m}\right)^{r}\|f\|_{W_{r}^{p}(u)}
$$

with $\mathcal{C} \neq \mathcal{C}(m, f)$. 
Let us consider, now, the $m$ th Fourier sum $S_{m}(w, f)$ of a function $f \in L_{u}^{p}$

$$
S_{m}(w, f)=\sum_{k=0}^{m-1} c_{k} p_{k}(w)
$$

where

$$
c_{k}=\int_{0}^{\infty} f(x) p_{k}(w, x) w(x) d x .
$$

In [12] the authors establish the following results.

Lemma 2.4. Let $1<p<+\infty, v^{\sigma}(x)=x^{\sigma}, w(x)=v^{\alpha}(x) e^{-x^{\beta}}$ and $u(x)=v^{\gamma}(x) e^{-x^{\beta} / 2}$. Then, for all functions $f \in L_{u}^{p}$, we have

$$
\left\|S_{m}\left(w, f_{j}\right) u\right\|_{p} \leq \mathcal{C} m^{1 / 3}\|f u\|_{p}, \quad \mathcal{C} \neq \mathcal{C}(m, f) .
$$

Moreover, for any $\bar{\theta} \in(0,1)$,

$$
\left\|S_{m}\left(w, f_{j}\right) u\right\|_{L^{p}\left(\left(0, \bar{\theta} a_{m}\right)\right)} \leq \mathcal{C}\left\|f_{j} u\right\|_{p}, \quad \mathcal{C} \neq \mathcal{C}(m, f)
$$

if and only if

$$
\frac{\alpha}{2}+\frac{1}{4}-\frac{1}{p}<\gamma<\frac{\alpha}{2}+\frac{3}{4}-\frac{1}{p}
$$

Let us note that conditions (2.13) are equivalent to

$$
\frac{v^{\gamma}}{\sqrt{v^{\alpha} \varphi}} \in L^{p}(0,1) \quad \text { and } \quad \sqrt{\frac{v^{\alpha}}{\varphi}} \frac{1}{v^{\gamma}} \in L^{q}(0,1)
$$

where $\frac{1}{p}+\frac{1}{q}=1$ and $\varphi(x)=\sqrt{x}$.

2.3. Approximations of the integral operator. Let us define the integral operator $K$ as

$$
(K f)(y)=\int_{0}^{\infty} k(x, y) f(x) w(x d x
$$


with $w(x)=x^{\alpha} e^{-x^{\beta}}, \alpha>-1, \beta>1 / 2$. Now, if $\theta \in(0,1)$ is fixed, let the integer $j=j(m)$, the function $\Phi_{j}$ is defined as in subsection 2.2. Then, we introduce the following operator

$$
(\widetilde{K} f)(y)=\int_{0}^{\infty}\left(k_{y}\right)_{j}(x) f(x) w(x) d x
$$

with $k(x, y)=k_{x}(y)=k_{y}(x),\left(k_{y}\right)_{j}=k_{y} \Phi_{j}$.

With $u(x)=x^{\gamma} e^{-x^{\beta} / 2}$, if the linear operator $K$ satisfies the condition

$$
\|K f\|_{W_{r}^{p}(u)} \leq \mathcal{C}\|f u\|_{p}, \quad r \in \mathbb{N}, r \geq 1
$$

for $1<p<+\infty$ and $\mathcal{C} \neq \mathcal{C}(f)$, then $K: L_{u}^{p} \rightarrow W_{r}^{p}(u)$ is bounded and is compact as a map of $L_{u}^{p}$ into $L_{u}^{p}$, and for equation (1.1) the Fredholm alternative is true.

In the following proposition we establish sufficient conditions on the kernel $k(x, y)$ which make (2.17) satisfied.

Proposition 2.5. Let $1<p<+\infty$. If $u(x)=x^{\gamma} e^{-x^{\beta} / 2}, \beta>1 / 2$, is such that

$$
\gamma<\alpha+1-\frac{1}{p}
$$

and if the kernel $k(x, y)$ verifies

$$
\int_{0}^{\infty}\left|\left\|k_{x}\right\|_{W_{r}^{p}(u)} \frac{w(x)}{u(x)}\right|^{q} d x<+\infty
$$

with $(1 / p)+(1 / q)=1$, then $(2.17)$ holds and also

$$
\|\widetilde{K} f\|_{W_{r}^{p}(u)} \leq \mathcal{C}\|f u\|_{p}
$$

Now let us define another approximating operator as follows

$$
\left(K_{m} f\right)(y)=L_{m+1}^{*}\left(w,(\widetilde{K} f)_{j} ; y\right)=\sum_{k=1}^{j} \tilde{l}_{k}(y)(\widetilde{K} f)\left(x_{k}\right)
$$


where $j=j(m),(\tilde{K} f)_{j}=(\tilde{K} f) \Phi_{j}$ and the interpolating operators $L_{m+1}^{*}(w)$ are as defined in subsection 2.2. The following proposition holds.

Proposition 2.6. Let $1<p<+\infty, v^{\sigma}(x)=x^{\sigma}$, and assume that $w(x)=v^{\alpha}(x) e^{-x^{\beta}}$ and $u(x)=v^{\gamma}(x) e^{-x^{\beta} / 2}$ satisfy (2.5) and (2.18). If the kernel $k(x, y)$ verifies condition (2.19) and

$$
\int_{0}^{\infty}\left|\left\|k_{y}\right\|_{W_{r}^{q}(w / u)} u(y)\right|^{p} d y<+\infty
$$

with $(1 / p)+(1 / q)=1$, then one has

$$
\left\|K-K_{m}\right\|_{L_{u}^{p} \rightarrow L_{u}^{p}}=\mathcal{O}\left(\left(\frac{\sqrt{a_{m}}}{m}\right)^{r}\right) .
$$

with the constant in $\mathcal{O}$ independent of $m$.

3. Numerical methods. In this section we propose numerical methods to construct sequences of polynomials which converge to the solution $f$ of integral equation (1.1) in some suitable weighted space $L_{u}^{p}$.

By defining operator $K$ as in (1.1), we can rewrite equation (1.1) as follows

$$
(I-K) f=g,
$$

where $I$ denotes the identity operator.

In order to describe a numerical method, let us introduce the subspace of $\mathbb{P}_{m}$ defined by

$\mathcal{P}_{m}=\left\{P \in \mathbb{P}_{m}: P(x)=q_{j-1}(x)\left(a_{m}-x\right) \prod_{i=j+1}^{m}\left(x-x_{i}\right), q_{j-1} \in \mathbb{P}_{j-1}\right\}$

and set

$$
f_{m}=\sum_{i=1}^{j} \frac{\tilde{l}_{i}(x)}{\mu_{i}} a_{i}, \quad g_{m}=\sum_{i=1}^{j} \frac{\tilde{l}_{i}(x)}{\mu_{i}} b_{i}
$$


where $\mu_{i}=\left(\Delta x_{i}\right)^{1 / p} u\left(x_{i}\right), \Delta x_{i}=x_{i+1}-x_{i}, a_{i}=f\left(x_{i}\right) \mu_{i}$, and $b_{i}=g\left(x_{i}\right) \mu_{i}$.

Obviously $f_{m}, g_{m} \in \mathcal{P}_{m}$ and $\left(I-K_{m}\right)\left(\mathcal{P}_{m}\right) \subset \mathcal{P}_{m}$, with $K_{m}$ defined as in (2.21).

Moreover, it is easy to verify that any polynomial $q_{m} \in \mathcal{P}_{m}$ has a unique representation as

$$
q_{m}(x)=\sum_{i=1}^{j} q_{m}\left(x_{i}\right) \tilde{l}_{i}(x) .
$$

Then our numerical method will consist of computing the solution $f_{m} \in \mathcal{P}_{m}$ of the finite dimensional equation

$$
\left(I-K_{m}\right) f_{m}=g_{m}
$$

Using previous results and standard arguments of functional analysis one can prove the following theorem.

Theorem 3.1. Let $u$ and $w$ be such that conditions (2.5) and (2.18) are fulfilled. Assume that $g \in W_{r}^{p}(u)$ and $k(x, y)$ satisfying (2.19) and (2.22). If $\operatorname{Ker}(I-K)=\{0\}$ in $L_{u}^{p}$. Then, for any sufficiently large $m$, equation (3.2) has a unique solution $f_{m}^{*} \in \mathcal{P}_{m}$ and, denoting by $f^{*}$ the solution of (3.1), one has

$$
\left\|\left(f^{*}-f_{m}^{*}\right) u\right\|_{p} \leq \mathcal{C}\left(\frac{\sqrt{a_{m}}}{m}\right)^{r}\|g\|_{W_{r}^{p}(u)},
$$

where $\mathcal{C}$ is independent of $m, f^{*}$ and $f_{m}^{*}$. Moreover,

$$
\left|\operatorname{cond}(I-K)-\operatorname{cond}\left(I-K_{m}\right)\right|=\mathcal{O}\left(\left(\frac{\sqrt{a_{m}}}{m}\right)^{r}\right),
$$

where the operator norm is that induced by the $L_{u}^{p}$-norm and cond $(T)=$ $\|T\| \cdot\left\|T^{-1}\right\|$ if $T: L_{u}^{p} \rightarrow L_{u}^{p}$ is an invertible operator.

In order to compute the approximate polynomial solution $f_{m}$ we replace in (3.2) $K_{m}, f_{m}$ and $g_{m}$ by their expressions, and we get the system of $j$ equations in the $j$ unknowns $a_{j}$

$$
a_{i}-\mu_{i}\left(\widetilde{K} f_{m}\right)\left(x_{i}\right)=b_{i}, \quad i=1, \ldots, j
$$


that, after simple calculations, becomes

$$
\begin{gathered}
\sum_{k=1}^{j}\left[\delta_{i k}-\frac{\mu_{i} \lambda_{k}(w)}{\mu_{k}\left(a_{m}-x_{k}\right)} S_{m}\left(w,\left(k_{x_{i}}\right)_{j}\left(a_{m}-\cdot\right) ; x_{k}\right)\right] a_{k}=b_{i} \\
i=1, \ldots, j
\end{gathered}
$$

with $\mu_{k}=\left(\Delta x_{k}\right)^{1 / p} u\left(x_{k}\right), k_{x}(y)=k(x, y), \lambda_{k}(w)$ the $k$ th Christoffel number with respect to the weight $w$ and $S_{m}(w, F)$ the $m$ th Fourier sum related to the function $F$ with respect to the orthonormal system $\left\{p_{m}(w)\right\}$.

System (3.5) is equivalent to equation (3.2) in the following sense: for every fixed $j$ the array $\left(\alpha_{1}, \ldots, \alpha_{j}\right) \in \mathbb{R}^{j}$ is a solution of system (3.5) if and only if

$$
f_{m}(y)=\sum_{i=1}^{j} \frac{\tilde{l}_{i}(y)}{\mu_{i}} \alpha_{i}
$$

is a solution of (3.2).

If we denote by $M_{j} \in \mathbb{R}^{j \times j}, j=j(m)$, the matrix of system (3.5), the following proposition holds.

Proposition 3.2. Under the assumptions of Theorem 1, the matrix $M_{j}$ of system (3.5) satisfies

$$
\text { cond }\left(M_{j}\right) \leq \mathcal{C} \text { cond }(I-K), \quad \mathcal{C} \neq \mathcal{C}(m)
$$

where cond $\left(M_{j}\right)$ is the condition number of $M_{j}$ with respect to the matrix norm induced by the vector p-norm.

We point out that, in order to construct an approximate solution $f_{m}$ of (3.1), we have to solve a linear system of $j$ equations in $j$ unknowns rather than a system of $m$ equations in $m$ unknowns, neglecting in this way $\left[\mathrm{Cm}^{2}\right](\mathcal{C}<1)$ terms.

Let us make a further remark. The numerical procedure described is, essentially, a projection method based on the projection operator $L_{m+1}^{*}(w)$ defined in (2.2). This Lagrange interpolation operator, we recall, is based on the $m$ zeros of the orthonormal polynomial $p_{m}(w)$ and on the additional node $a_{m}$. To include $a_{m}$ in the set of the 
interpolation points permits us to guarantee the boundedness of the projector itself. Moreover, for reasons of computational economy, we really use only polynomials interpolating truncated functions.

Now let us observe that some computational efforts are necessary to evaluate the entries of matrix $M_{j}$, since

$$
\frac{\lambda_{k}(w)}{a_{m}-x_{k}} S_{m}\left(w,\left(k_{x_{i}}\right)_{j}\left(a_{m}-\cdot\right) ; x_{k}\right)=\left(\tilde{K} \tilde{l}_{k}\right)\left(x_{i}\right)
$$

and, in the general case $w(x)=x^{\alpha} e^{-x^{\beta}}, \beta>1 / 2$, recurrence relations holding for orthonormal polynomials $\left\{p_{m}(w)\right\}$ are not available. Nevertheless, for $\beta=1$ (Laguerre case) and kernels of convolution type $(k(x, y)=k(|x-y|))$, one can use the recurrence relation of the Laguerre polynomials and the elements of $M_{j}$ becomes more easily computable. However such computational difficulties naturally appear in the case of kernels and weights which are not standard.

Then it is useful to show that, under additional conditions on the weights $w$ and $u$ and on the kernel $k(x, y)$, we can replace system (3.5) by the following more simple one

$$
\sum_{k=1}^{j}\left[\delta_{i k}-\frac{\mu_{i} \lambda_{k}(w)}{\mu_{k}} k\left(x_{k}, x_{i}\right)\right] v_{k}=b_{i}, \quad i=1, \ldots, j
$$

with $\mu_{i}=\left(\Delta x_{i}\right)^{1 / p} u\left(x_{i}\right)$. Moreover, by using a Mathematica package in [2] to compute the quantities $\lambda_{k}(w)$ and $x_{k}$, the construction of system (3.7) turns out simple. The following theorem holds true.

Theorem 3.3. Let $w(x)=x^{\alpha} e^{-x^{\beta}}$ and $u(x)=x^{\gamma} e^{-x^{\beta} / 2}$ be such that

$$
\frac{\alpha}{2}+\frac{1}{4}-\frac{1}{p}<\gamma<\frac{\alpha}{2}+\frac{3}{4}-\frac{1}{p}
$$

and (2.18) are fulfilled. Assume $g \in W_{r}^{p}(u)$ and $k(x, y)$ satisfying (2.19), (2.22) and the further condition

$$
\int_{0}^{\infty}\left|\frac{\partial^{r}}{\partial y^{r}}\left(\left\|k_{y}\right\|_{W_{r}^{q}(w / u)}\right) \varphi^{r}(y) u(y)\right|^{p} d y<+\infty .
$$


If $\operatorname{Ker}(I-K)=\{0\}$ in $L_{u}^{p}$, then, for any sufficiently large $m$, system (3.7) has a unique solution $\left(v_{1}^{*}, \ldots, v_{j}^{*}\right)$ and the corresponding polynomial

$$
f_{m}^{* *}=\sum_{i=1}^{j} \frac{\tilde{l}_{i}}{\mu_{i}} v_{i}^{*} \in \mathcal{P}_{m}
$$

satisfies the estimate

$$
\left\|\left(f^{*}-f_{m}^{* *}\right) u\right\|_{p} \leq \mathcal{C}\left(\frac{\sqrt{a_{m}}}{m}\right)^{r}\|g\|_{W_{r}^{p}(u)}
$$

where $f^{*}$ is the solution of (3.1) and $\mathcal{C}$ is independent of $m, f^{*}$ and $f_{m}^{* *}$. Moreover, the matrix $M_{j}^{*}$ of system (3.7), satisfies

$$
\operatorname{cond}\left(M_{j}^{*}\right) \leq \mathcal{C} \text { cond }(I-K), \quad \mathcal{C} \neq \mathcal{C}(m)
$$

where cond $\left(M_{j}^{*}\right)$ is the condition number of $M_{j}^{*}$ with respect to the matrix norm induced by the vector p-norm.

Let us make two remarks.

Remark 1. Since

$$
\lambda_{m}\left(u^{p}, x_{i}\right) \sim\left(\Delta x_{i}\right) u^{p}\left(x_{i}\right)
$$

(see $[\mathbf{9}]$ ) with

$$
\lambda_{m}\left(u^{p}, x\right)=\left[\sum_{k=0}^{m-1} p_{k}^{2}\left(u^{p}, x\right)\right]^{-1},
$$

the $m$ th Christoffel function related to the weight $u^{p}$ and the constants in $\sim$ independent of $m$ and $i$, alternative numerical procedures can be obtained by replacing $\mu_{i}=\left(\Delta x_{i}\right)^{1 / p} u\left(x_{i}\right)$ everywhere by $\nu_{i}=$ $\lambda_{m}^{1 / p}\left(u^{p}, x_{i}\right)$. In this way the polynomial solution is given by

$$
f_{m}(y)=\sum_{i=1}^{j} \frac{\tilde{l}_{i}(x)}{\nu_{i}} A_{i},
$$


with $\left(A_{1}, A_{2}, \ldots, A_{m}\right)$ obtained as a solution of the following system

$$
\begin{gathered}
\sum_{k=1}^{j}\left[\delta_{i k}-\frac{\nu_{i} \lambda_{k}(w)}{\nu_{k}\left(a_{m}-x_{k}\right)} S_{m}\left(w, k_{x_{i}}\left(a_{m}-\cdot\right) ; x_{k}\right)\right] A_{k}=B_{i}, \\
i=1, \ldots, j,
\end{gathered}
$$

or, in the case of smooth kernels,

$$
\sum_{k=1}^{j}\left[\delta_{i k}-\frac{\nu_{i} \lambda_{k}(w)}{\nu_{k}} k\left(x_{k}, x_{i}\right)\right] A_{k}=B_{i}, \quad i=1, \ldots, j
$$

with $B_{i}=g\left(x_{i}\right) \lambda_{m}^{1 / p}\left(u^{p}, x_{i}\right)$.

All the previous results hold again, but the computation of the coefficients of the new systems is more difficult because it requires evaluating the orthonormal polynomials $p_{k}\left(u^{p}, x\right), k=0,1, \ldots, m-1$. Nevertheless, if, in particular, $p=2$, and $u(x)=\sqrt{w(x)}$, i.e., $\gamma=\alpha / 2$, one has $\lambda_{m}\left(u^{p}, x_{i}\right)=\lambda_{i}(w)$.

Remark 2. The conditions previously assumed both on the kernel $k(x, y)$ and on the right-hand side function $g(y)$ can be relaxed if we replace the Sobolev-type spaces (that we use in order to simplify the proofs) by Zygmund-type ones defined in subsection 2.1.

Nyström method. A direct consequence of the last theorem is the stability of the Nyström method based on a truncated Gaussian quadrature rule (see $[\mathbf{1 0}]$ ). In fact, if we approximate the integral $(K f)(y)$ by

$$
\begin{aligned}
\left(\bar{K}_{m} f\right)(y) & =\int_{0}^{\infty} L_{m+1}^{*}\left(w,(k(\cdot, y) f)_{j} ; x\right) w(x) d x \\
& =\sum_{k=1}^{j} \lambda_{k}(w) \frac{k\left(x_{k}, y\right)}{\lambda_{m}^{1 / p}\left(u^{p}, x_{k}\right)} A_{k},
\end{aligned}
$$

with $A_{k}=\lambda_{m}^{1 / p}\left(u^{p}, x_{k}\right) f\left(x_{k}\right)$, we have to solve the following equation

$$
\bar{f}_{m}(y)-\sum_{k=1}^{j} \lambda_{k}(w) \frac{k\left(x_{k}, y\right)}{\lambda_{m}^{1 / p}\left(u^{p}, x_{k}\right)} A_{k}=g(y) .
$$


By multiplying the last equation, on both sides, by the Christoffel function $\lambda_{m}^{1 / p}\left(u^{p}, y\right)$ and collocating in the points $x_{1}, \ldots, x_{j}$, we get the system (3.15) which is, as we have said before, well conditioned. Then, we can construct the approximating solution of integral equation (3.1) by means of the Nyström interpolation formula

$$
\bar{f}_{m}(y)=g(y)+\sum_{k=1}^{j} \lambda_{k}(w) \frac{k\left(x_{k}, y\right)}{\lambda_{m}^{1 / p}\left(u^{p}, x_{k}\right)} \bar{A}_{k}
$$

corresponding to the solution $\left(\bar{A}_{1}, \ldots, \bar{A}_{j}\right)$ of $(3.15)$.

By virtue of (3.12), we can obtain the Nyström interpolating function also by applying the formula

$$
\bar{f}_{m}(y)=g(y)+\sum_{k=1}^{j} \lambda_{k}(w) \frac{k\left(x_{k}, y\right)}{\left(\Delta x_{k}\right)^{1 / p} u\left(x_{k}\right)} \bar{a}_{k} .
$$

with $\left(\bar{a}_{1}, \ldots, \bar{a}_{j}\right)$ a solution of system (3.7). The sequence $\bar{f}_{m}$ converges to the exact solution $f$ of (3.1) in $L_{u}^{p}$ as stated in the following theorem.

Theorem 3.4. Under the assumptions of Theorem 3.3, one has

$$
\left\|\left(f^{*}-\bar{f}_{m}\right) u\right\|_{p} \leq \mathcal{C}\left(\frac{\sqrt{a_{m}}}{m}\right)^{r}\left\|f^{*}\right\|_{W_{r}^{p}(u)} .
$$

Let us observe that the error is of the same order of best approximation in the considered space $L_{u}^{p}$ for functions belonging to $W_{r}^{p}(u)$ (see estimate (2.1)).

4. Numerical Examples. In this section we show some examples of Fredholm integral equations solved by using the numerical procedure described in the previous section.

We evaluate the solution $\bar{f}_{m} u$ in some points and the condition number

$$
\operatorname{cond}_{2}\left(M_{j}^{*}\right)=\left\|M_{j}^{*}\right\|_{2}\left\|\left(M_{j}^{*}\right)^{-1}\right\|_{2}
$$

of the linear system (3.7). Unfortunately we are not able to compute $\operatorname{cond}_{p}\left(M_{j}^{*}\right)$ when $p \notin\{1,2\}$. 
Finally we represent the graphic of the function $\bar{f}_{m}(x) u(x)$. We point out that the zeros $x_{1}, \ldots, x_{m}$ of $p_{m}(w)$ and the Christoffel numbers $\lambda_{1}(w), \ldots, \lambda_{m}(w)$, for a generalized Laguerre weight $w(x)=x^{\alpha} e^{-x^{\beta}}$, $\alpha>-1, \beta>1 / 2$, are computed by using a Mathematica package appearing in $[\mathbf{2}]$.

Example 4.1. Consider the integral equation

$$
f(y)-\int_{0}^{+\infty} x e^{-x} y^{3} f(x) \sqrt{x} e^{-x^{5 / 2}} d x=e^{y}-\frac{2}{5} y^{3} .
$$

Let $k(x, y)=x y^{3} e^{-x}$ and $w(x)=x^{1 / 2} e^{-x^{5 / 2}}$. We study the equation in the space $L_{u}^{p}$ with $p=2$ and the weight $u(x)=x^{1 / 8} e^{-\left(x^{5 / 2}\right) / 2}$, chosen according to conditions (2.5) and (2.18). We fix $\theta=0.6$ and construct the approximate solution $\bar{f}_{m}$ by solving system (3.7) and using the Nyström interpolation formula (3.17). Since, in this case, the exact solution is known, $f(x)=e^{x}$, we report in Table 4.1.1 the weighted error $\left|f(y)-\bar{f}_{m}(y)\right| u(y)$ at some points. As one can note, we get results with machine precision in double arithmetic with small values of $m$. Table 4.1.2 shows that the condition number $\operatorname{cond}_{2}\left(M_{j}^{*}\right)$ of the matrix $M_{j}^{*}$ (of order $j$ ) is very small.

\begin{tabular}{|l|l|l|l|l|}
\hline \multicolumn{5}{|c|}{ Table 4.1.1 } \\
\hline \multicolumn{5}{|c|}{$\left|f(y)-\bar{f}_{m}(y)\right| u(y)$} \\
\hline$m$ & $j$ & $y=0.5$ & $y=1$ & $y=1.5$ \\
\hline 16 & 10 & $2.305069283359273 \mathrm{e}-004$ & $1.332422784794840 \mathrm{e}-003$ & $1.966468727766735 \mathrm{e}-003$ \\
\hline 32 & 19 & $1.040700395638083 \mathrm{e}-005$ & $6.015666987924107 \mathrm{e}-005$ & $8.878278834201225 \mathrm{e}-005$ \\
\hline 64 & 39 & $5.812201830934782 \mathrm{e}-010$ & $3.359685862847073 \mathrm{e}-009$ & $4.958424115741877 \mathrm{e}-009$ \\
\hline 128 & 78 & 0. & 0. & $2.220446049250313 \mathrm{e}-016$ \\
\hline 256 & 156 & 0. & 0. & $4.440892098500626 \mathrm{e}-016$ \\
\hline
\end{tabular}

\begin{tabular}{|l|l|l|}
\hline \multicolumn{3}{|c|}{ Table 4.1 .2} \\
\hline$m$ & $j$ & $\operatorname{cond}_{2}\left(M_{j}^{*}\right)$ \\
\hline 16 & 10 & $1.217475911175006 \mathrm{e}+000$ \\
\hline 32 & 19 & $1.229579009747493 \mathrm{e}+000$ \\
\hline 64 & 39 & $1.231447708039921 \mathrm{e}+000$ \\
\hline 128 & 78 & $1.231977147831094 \mathrm{e}+000$ \\
\hline 256 & 156 & $1.232305170639684 \mathrm{e}+000$ \\
\hline
\end{tabular}




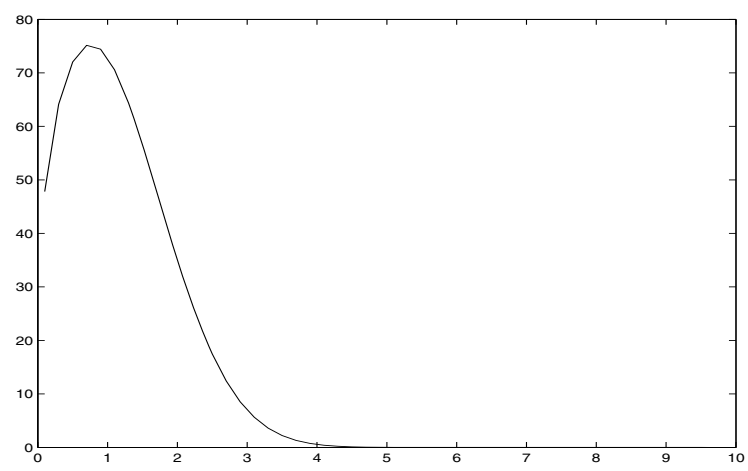

Figure 1. Graphic of $\bar{f}_{m}(x) u(x)$ with $m=256$

Example 4.2. Let

$$
f(y)-\frac{1}{2} \int_{0}^{+\infty}(2 x+y) e^{-x y} f(x) \frac{e^{-x^{2}}}{\sqrt[4]{x}} d x=e^{y+3} .
$$

In this case the kernel is $k(x, y)=(1 / 2)(2 x+y) e^{-x y}$ and the weight $w(x)=x^{-1 / 4} e^{-x^{2}}$. We study the integral equation in $L_{u}^{p}$ with $p=4$ and $u(x)=x^{1 / 4} e^{-x^{2} / 2}$. Fixing $\theta=0.7$, we get the results shown in Tables 4.2.1 and 4.2.2.

\begin{tabular}{|c|c|c|c|c|}
\hline \multicolumn{5}{|c|}{ Table 4.2 .1} \\
\hline \multicolumn{5}{|c|}{$\bar{f}_{m}(y) u(y)$} \\
\hline$m$ & $j$ & $y=1$ & $y=3$ & $y=5$ \\
\hline 16 & 10 & $\mathbf{7 . 2 7 9 1 2 0 0 4 9 0 0 0 0 6 3 e + 0 0 1}$ & 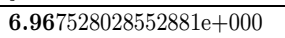 & $\mathbf{1 . 7 0 5 7 3 7 6 7 4 8 2 6 7 2 2 \mathrm { e } - 0 0 2}$ \\
\hline 32 & 21 & $\mathbf{7 . 2 8 5 4 8 9 6 7 0 7 0 5 2 0 4 \mathrm { e } + 0 0 1}$ & 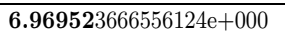 & $\mathbf{1 . 7 0 5 8 2 9 3 3 9 5 1 9 2 6 0 \mathrm { e } - 0 0 2}$ \\
\hline 64 & 42 & 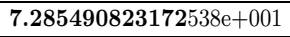 & $6.969524075559379 \mathrm{e}+000$ & $1.705829358921819 \mathrm{e}-002$ \\
\hline 128 & 83 & $\mathbf{7 . 2 8 5 4 9 0 8 2 3 1 7 2 8 8 9 e + 0 0 1}$ & $6.969524075559513 \mathrm{e}+000$ & $1.705829358921826 \mathrm{e}-002$ \\
\hline 256 & 166 & 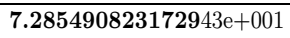 & $6.969524075559538 \mathrm{e}+000$ & $1.705829358921827 \mathrm{e}-002$ \\
\hline
\end{tabular}

\begin{tabular}{|l|l|l|}
\hline \multicolumn{3}{|c|}{ Table 4.2.2 } \\
\hline$m$ & $j$ & $\operatorname{cond}_{2}\left(M_{j}^{*}\right)$ \\
\hline 16 & 10 & $3.310708752025420 \mathrm{e}+000$ \\
\hline 32 & 21 & $3.360568961784741 \mathrm{e}+000$ \\
\hline 64 & 42 & $3.400065377210209 \mathrm{e}+000$ \\
\hline 128 & 78 & $3.432531945475642 \mathrm{e}+000$ \\
\hline 256 & 166 & $3.458917389149575 \mathrm{e}+000$ \\
\hline
\end{tabular}


Example 4.3. Consider the integral equation

$$
f(y)-\frac{3}{4} \int_{0}^{+\infty} \frac{f(x)}{x+y+2} x^{2} e^{-x} d x=y^{\frac{3}{2}}
$$

where

$$
k(x, y)=\frac{3}{4(x+y+2)} \text { and } w(x)=x^{2} e^{-x} .
$$

Choosing $u(x)=x^{3 / 4} e^{-x / 2}$, satisfying conditions (2.5) and (2.18), $\theta=0.7$ and $p=3$ we obtain the results presented in the following

\begin{tabular}{|c|c|c|c|c|}
\hline \multicolumn{5}{|c|}{ Table 4.3 .1} \\
\hline \multicolumn{5}{|c|}{$\bar{f}_{m}(y) u(y)$} \\
\hline$m$ & $j$ & $y=0.5$ & $y=1.5$ & $y=2.5$ \\
\hline 16 & 7 & $\mathbf{9 . 7 0 9 2 2 1 7 2 0 5 9 1 0 0 0 \mathrm { e } - 0 0 1}$ & 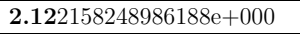 & 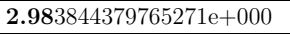 \\
\hline 32 & 16 & 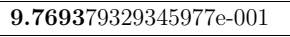 & 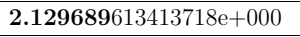 & $2.989995980426683 \mathrm{e}+000$ \\
\hline 64 & 32 & 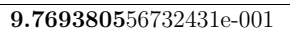 & 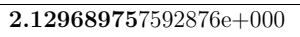 & 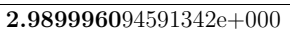 \\
\hline 128 & 65 & $\mathbf{9 . 7 6 9 3 8 0 5 7 7 2 4 3 9 2 9 \mathrm { e } - 0 0 1}$ & 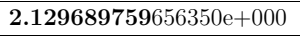 & 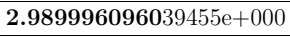 \\
\hline 256 & 131 & 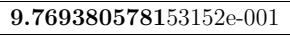 & $\mathbf{2 . 1 2 9 6 8 9 7 5 9 7 4 8 1 6 6 e + 0 0 0}$ & $2.989996096104016 \mathrm{e}+000$ \\
\hline 512 & 264 & 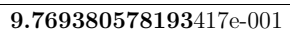 & $2.129689759752238 \mathrm{e}+000$ & $2.989996096106883 \mathrm{e}+000$ \\
\hline
\end{tabular}
tables.

\begin{tabular}{|l|l|l|}
\hline \multicolumn{3}{|c|}{ Table 4.3.2 } \\
\hline$m$ & $j$ & $\operatorname{cond}_{2}\left(M_{j}^{*}\right)$ \\
\hline 16 & 7 & $1.296060849364210 \mathrm{e}+000$ \\
\hline 32 & 16 & $1.296261865762703 \mathrm{e}+000$ \\
\hline 64 & 32 & $1.295775625957592 \mathrm{e}+000$ \\
\hline 128 & 65 & $1.295257961055335 \mathrm{e}+000$ \\
\hline 256 & 131 & $1.294825106535888 \mathrm{e}+000$ \\
\hline 512 & 264 & $1.294491673546656 \mathrm{e}+000$ \\
\hline
\end{tabular}

\section{Proofs of the main results.}

Proof of Proposition 2.5. We have to estimate both $\|(K f) u\|_{p}$ and $\left\|(K f)^{(r)} \varphi^{r} u\right\|_{p}$. By applying the Minkowski and Hölder inequalities 


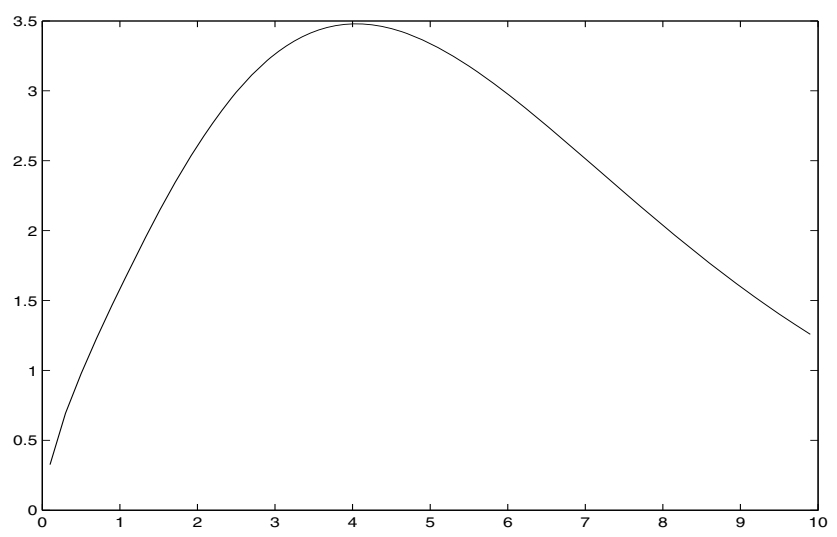

Figure 2. Graphic of $\bar{f}_{m}(x) u(x)$ with $m=512$

and taking into account (2.19), we have

$$
\begin{aligned}
\|(K f) u\|_{p} & \leq \int_{0}^{\infty}|f(x)| w(x)\left(\int_{0}^{\infty}|k(x, y) u(y)|^{p} d y\right)^{1 / p} d x \\
& \leq \int_{0}^{\infty}|f(x)| u(x) \frac{w(x)}{u(x)}\left\|k_{x} u\right\|_{p} d x \\
& \leq\|f u\|_{p}\left(\int_{0}^{\infty}\left|\left\|k_{x} u\right\|_{p} \frac{w(x)}{u(x)}\right|^{q} d x\right)^{1 / q} \leq \mathcal{C}\|f u\|_{p}
\end{aligned}
$$

and

$$
\begin{aligned}
\|(K f)^{(r)} & \varphi^{r} u \|_{p} \\
& \leq \int_{0}^{\infty}|f(x)| w(x)\left(\int_{0}^{\infty}\left|\frac{\partial^{r}}{\partial y^{r}} k(x, y) \varphi^{r}(y) u(y)\right|^{p} d y\right)^{1 / p} d x \\
& \leq \int_{0}^{\infty}|f(x)| u(x) \frac{w(x)}{u(x)}\left\|k_{x}^{(r)} \varphi^{r} u\right\|_{p} d x \\
& \leq\|f u\|_{p}\left(\int_{0}^{\infty}\left|\left\|k_{x}^{(r)} \varphi^{r} u\right\|_{p} \frac{w(x)}{u(x)}\right|^{q} d x\right)^{1 / q} \leq \mathcal{C}\|f u\|_{p} .
\end{aligned}
$$

Then (2.17) holds. The proof of (2.20) is similar. 
Now we establish a lemma which we will use later. Let $\theta \in(0,1)$ be fixed, and let $m$ be a positive sufficiently large integer. Let $j$ and the corresponding function $\Phi_{j}$ be as defined in Section 2, and let

$$
M=\left[\left(\frac{\theta}{\theta+1}\right)^{\beta} m\right] \sim m .
$$

Then one can prove (see $[\mathbf{1 4}]$ ) the following result.

Lemma 5.1. Let $1 \leq p \leq \infty$ and $f \in L_{u}^{p}$. Then, for any sufficiently large $m$, we have

$$
\left\|\left(f-f_{j}\right) u\right\|_{p} \leq \mathcal{C}\left[E_{M}(f)_{u, p}+e^{-A m}\|f u\|_{p}\right]
$$

with the constants $\mathcal{C}$ and $A$ positive and independent of $m$ and $f$.

Proof of Proposition 2.6. We have

$\left\|\left[\left(K-K_{m}\right) f\right] u\right\|_{p} \leq\|[(K-\widetilde{K}) f] u\|_{p}+\left\|\left[\tilde{K} f-L_{m+1}^{*}\left(w,(\tilde{K} f)_{j}\right)\right] u\right\|_{p}$.

Let us estimate $\|[(K-\widetilde{K}) f] u\|_{p}$. To this end we apply at first (5.1) and later $(2.1)$ to the function $k_{y}(x)$ and obtain

$$
\begin{aligned}
\left\|\left[k_{y}-\left(k_{y}\right)_{j}\right] \frac{w}{u}\right\|_{q} & \leq \mathcal{C}\left[E_{M}\left(k_{y}\right)_{(w / u), q}+e^{-A m}\left\|k_{y} \frac{w}{u}\right\|_{q}\right] \\
& \leq \mathcal{C}\left(\frac{\sqrt{a_{m}}}{m}\right)^{r}\left\|k_{y}\right\|_{W_{q}^{r}(w / u)} .
\end{aligned}
$$

Then we can write, by Hölder's inequality,

$$
\begin{aligned}
\|[(K-\widetilde{K}) f] u\|_{p} \\
\quad=\left(\int_{0}^{\infty}\left|\int_{0}^{\infty}\left[k_{y}(x)-\left(k_{y}\right)_{j}(x)\right] f(x) w(x) d x u(y)\right|^{p} d y\right)^{1 / p} \\
\leq\|f u\|_{p}\left(\int_{0}^{\infty}\left|\left\|\left[k_{y}-\left(k_{y}\right)_{j}\right] \frac{w}{u}\right\|_{q} u(y)\right|^{p} d y\right)^{1 / p} \\
\leq \mathcal{C}\left(\frac{\sqrt{a_{m}}}{m}\right)^{r}\|f u\|_{p}\left(\int_{0}^{\infty}\left|\left\|k_{y}\right\|_{W_{q}^{r}(w / u)} u(y)\right|^{p} d y\right)^{1 / p} \\
\leq \mathcal{C}\left(\frac{\sqrt{a_{m}}}{m}\right)^{r}\|f u\|_{p}
\end{aligned}
$$

by the assumptions. 
It remains to estimate $\left\|\left[\tilde{K} f-L_{m+1}^{*}\left(w,(\widetilde{K} f)_{j}\right)\right] u\right\|_{p}$. Taking into account (2.20) and using (2.10) applied to $\widetilde{K} f$, we obtain

$$
\begin{aligned}
\left\|\left[\widetilde{K} f-L_{m+1}^{*}\left(w,(\widetilde{K} f)_{j}\right)\right] u\right\|_{p} & \leq \mathcal{C}\left(\frac{\sqrt{a_{m}}}{m}\right)^{r}\|(\widetilde{K} f) u\|_{p} \\
& \leq \mathcal{C}\left(\frac{\sqrt{a_{m}}}{m}\right)^{r}\|f u\|_{p} .
\end{aligned}
$$

Then, the assertion follows.

Proof of Theorem 3.1. Let us observe that, under our assumptions, (2.23) holds and, by applying (2.10) to the function $g$, we also have

$$
\left\|\left(g-g_{m}\right) u\right\|_{p} \leq \mathcal{C}\left(\frac{\sqrt{a_{m}}}{m}\right)^{r}\|g\|_{W_{r}^{p}(u)} .
$$

By standard arguments (see [6], Theorem 2.1), the assertion follows.

To prove Proposition 3.2 we shall use the following result proved in [15].

Lemma 5.2. Let $0<\theta<\theta_{1}<1$, and let $1 \leq p<+\infty$. Then, for an arbitrary polynomial $P \in \mathbb{P}_{l m}$ (with l a fixed integer), we have

$$
\left(\sum_{k=1}^{j} \Delta x_{k}|P u|^{p}\left(x_{k}\right)\right)^{1 / p} \leq \mathcal{C}\left(\int_{x_{1}}^{\theta_{1} a_{m}}|P u|^{p}(x) d x\right)^{1 / p}
$$

where $\Delta x_{k}=x_{k+1}-x_{k}$ and $\mathcal{C}$ is a positive constant independent of $m$ and $P$.

Proof of Proposition 3.2. In the sequel let us denote by $\|\mathbf{c}\|_{l_{p}}=$ $\left(\sum_{k=1}^{j}\left|c_{k}\right|^{p}\right)^{1 / p}$ the $l_{p}$-norm of a vector $\mathbf{c}=\left(c_{1}, c_{2}, \ldots, c_{j}\right) \in \mathbb{R}^{j}$.

Let $\mathbf{a}=\left(a_{1}, a_{2}, \ldots, a_{j}\right) \in \mathbb{R}^{j}$ be an arbitrary vector and let $\mathbf{b}=$ $\left(b_{1}, b_{2}, \ldots, b_{j}\right)$ be given by $\mathbf{b}=M_{j} \mathbf{a}$. If we define

$$
F_{m}=\sum_{k=1}^{j} \frac{\widetilde{l_{k}}}{\mu_{k}} a_{k}, \quad \mu_{k}=\left(\Delta x_{k}\right)^{1 / p} u\left(x_{k}\right),
$$


then $F_{m}$ satisfies the equation $\left(I-K_{m}\right) F_{m}=G_{m}$ if and only if

$$
G_{m}=\sum_{k=1}^{j} \frac{\widetilde{l}_{k}}{\mu_{k}} b_{k}
$$

Let us note that, for $k=1, \ldots, j$, we have $a_{k}=F_{m}\left(x_{k}\right) \mu_{k}$ and $b_{k}=G_{m}\left(x_{k}\right) \mu_{k}$. Then, by applying at first (5.2) and later (2.6) we have

$$
\begin{aligned}
\left\|M_{j} \mathbf{a}\right\|_{l_{p}} & =\|\mathbf{b}\|_{l_{p}}=\left(\sum_{k=0}^{j} \Delta x_{k}\left|G_{m}\left(x_{k}\right) u\left(x_{k}\right)\right|^{p}\right)^{1 / p} \\
& \leq \mathcal{C}\left(\int_{x_{1}}^{\theta_{1} a_{m}}\left|G_{m}(x) u(x)\right|^{p} d x\right)^{1 / p} \\
& \leq \mathcal{C}\left(\int_{0}^{+\infty}\left|G_{m}(x) u(x)\right|^{p} d x\right)^{1 / p} \\
& \leq \mathcal{C}\left\|I-K_{m}\right\|\left\|F_{m} u\right\|_{p} \\
& \leq \mathcal{C}\left\|I-K_{m}\right\|\left(\sum_{k=0}^{j} \Delta x_{k}\left|F_{m}\left(x_{k}\right) u\left(x_{k}\right)\right|^{p}\right)^{1 / p} \\
& =\mathcal{C}\left\|I-K_{m}\right\|\|\mathbf{a}\|_{l_{p}},
\end{aligned}
$$

where $\left\|I-K_{m}\right\|=\left\|I-K_{m}\right\|_{L_{u}^{p} \rightarrow L_{u}^{p}}$ and $\mathcal{C} \neq \mathcal{C}(m)$. Then it follows

$$
\left\|M_{j}\right\| \leq \mathcal{C}\left\|I-K_{m}\right\|
$$

with $\left\|M_{j}\right\|=\left\|M_{j}\right\|_{l_{p} \rightarrow l_{p}}$.

Now let $\mathbf{b}=\left(b_{1}, b_{2}, \ldots, b_{j}\right)$ be an arbitrary vector in $\mathbb{R}^{j}, \mathbf{a}=$ $\left(a_{1}, a_{2}, \ldots, a_{j}\right)$ defined by $\mathbf{a}=M_{j}^{-1} \mathbf{b}$ and $G_{m}$ as in (5.4). Then $\left(I-K_{m}\right) F_{m}=G_{m}$ if and only if $F_{m}$ is as in (5.3). By (5.2) and 
(2.6) again, we get

$$
\begin{aligned}
\left\|M_{j}^{-1} \mathbf{b}\right\|_{l_{p}} & =\|\mathbf{a}\|_{l_{p}}=\left(\sum_{k=0}^{j} \Delta x_{k}\left|F_{m}\left(x_{k}\right) u\left(x_{k}\right)\right|^{p}\right)^{1 / p} \\
& \leq \mathcal{C}\left(\int_{x_{1}}^{\theta_{1} a_{m}}\left|F_{m}(x) u(x)\right|^{p} d x\right)^{1 / p} \\
& \leq \mathcal{C}\left(\int_{0}^{+\infty}\left|F_{m}(x) u(x)\right|^{p} d x\right)^{1 / p} \\
& \leq \mathcal{C}\left\|\left(I-K_{m}\right)^{-1}\right\|\left\|G_{m} u\right\|_{p} \\
& \leq \mathcal{C}\left\|\left(I-K_{m}\right)^{-1}\right\|\left(\sum_{k=0}^{j} \Delta x_{k}\left|G_{m}\left(x_{k}\right) u\left(x_{k}\right)\right|^{p}\right)^{1 / p} \\
& =\mathcal{C}\left\|\left(I-K_{m}\right)^{-1}\right\|\|\mathbf{b}\|_{l_{p}},
\end{aligned}
$$

where $\left\|\left(I-K_{m}\right)^{-1}\right\|=\left\|\left(I-K_{m}\right)^{-1}\right\|_{L_{u}^{p} \rightarrow L_{u}^{p}}$ and $\mathcal{C} \neq \mathcal{C}(m)$. Therefore, set $\left\|M_{j}^{-1}\right\|=\left\|M_{j}^{-1}\right\|_{l_{p} \rightarrow l_{p}}$,

$$
\left\|M_{j}^{-1}\right\| \leq \mathcal{C}\left\|\left(I-K_{m}\right)^{-1}\right\| .
$$

Combining (5.5) and (5.6) and, moreover, taking into account (3.4), we have

$\operatorname{cond}\left(M_{j}\right) \leq \mathcal{C} \operatorname{cond}\left(I-K_{m}\right)=\mathcal{C} \operatorname{cond}(I-K)+O\left(\left(\frac{\sqrt{a_{m}}}{m}\right)^{r}\right)$.

Proof of Theorem 3.3. Denote by $M_{j} \mathbf{a}=\mathbf{b}$ the system (3.5) and by $M_{j}^{*} \alpha=\mathbf{b}$ the system (3.7), respectively. Since, for sufficiently large $m$ (say $\left.m>m_{0}\right) M_{j}^{-1}$ exists, the identity

$$
M_{j}^{*}=M_{j}\left[I_{j}+M_{j}^{-1}\left(M_{j}^{*}-M_{j}\right)\right]
$$

holds true, with $I_{j}$ the identity matrix of dimension $j$. If we set

$$
M_{j}-M_{j}^{*}=E_{j}=\left(\varepsilon_{i k}\right)_{i, k=1, \ldots, j},
$$


we have

$$
\begin{aligned}
\varepsilon_{i k} & =\frac{\mu_{i} \lambda_{k}}{\mu_{k}\left(a_{m}-x_{k}\right)} \\
& \times\left[S_{m}\left(w,\left(k_{x_{i}}\right)_{j}\left(a_{m}-\cdot\right) ; x_{k}\right)-L_{m+1}^{*}\left(w,\left(k_{x_{i}}\right)_{j}\left(a_{m}-\cdot\right) ; x_{k}\right)\right]
\end{aligned}
$$

where, we recall, $\mu_{i}=\left(\Delta x_{i}\right)^{1 / p} u\left(x_{i}\right)$.

If we proved

$$
\left\|E_{j}\right\|=\mathcal{O}\left(\left(\frac{\sqrt{a_{m}}}{m}\right)^{r}\right),
$$

then, by applying the Neumann series argument, we can deduce that $\left(M_{j}^{*}\right)^{-1}$ also exists and

$$
\lim _{m} \frac{\operatorname{cond}\left(M_{j}^{*}\right)}{\operatorname{cond}\left(M_{j}\right)} \leq 1
$$

On the other hand, using the identity

$$
M_{j}=M_{j}^{*}\left[I_{j}+\left(M_{j}^{*}\right)^{-1}\left(M_{j}-M_{j}^{*}\right)\right]
$$

one can obtain, in the same way,

$$
\lim _{m} \frac{\operatorname{cond}\left(M_{j}\right)}{\operatorname{cond}\left(M_{j}^{*}\right)} \leq 1,
$$

and consequently,

$$
\lim _{m} \frac{\operatorname{cond}\left(M_{j}^{*}\right)}{\operatorname{cond}\left(M_{j}\right)}=1 .
$$

Combining (5.8) and (3.6), we get (3.11).

Moreover, as a consequence, if $\mathbf{a}^{*}=\left(a_{1}^{*}, \ldots, a_{j}^{*}\right)$ is the unique solution of system (3.5) and $\mathbf{v}^{*}=\left(v_{1}^{*}, \ldots, v_{j}^{*}\right)$ is the unique solution of system (3.7), then it results

$$
\frac{\left\|\mathbf{a}^{*}-\mathbf{v}^{*}\right\|_{l_{p}}}{\left\|\mathbf{a}^{*}\right\|_{l_{p}}} \leq \frac{\left\|M_{j}^{-1}\right\|\left\|E_{j}\right\|}{1-\left\|M_{j}^{-1}\right\|\left\|E_{j}\right\|}=\mathcal{O}\left(\left(\frac{\sqrt{a_{m}}}{m}\right)^{r}\right)
$$


since (see the proof of Proposition 3.2)

$$
\left\|M_{j}^{-1}\right\| \leq \mathcal{C}\left\|\left(I-K_{m}\right)^{-1}\right\| \leq C\left\|(I-K)^{-1}\right\|,
$$

for sufficiently large $m$. To prove (3.10) we use the following inequality

$$
\left\|\left(f^{*}-f_{m}^{* *}\right) u\right\|_{p} \leq\left\|\left(f^{*}-f_{m}^{*}\right) u\right\|_{p}+\left\|\left(f_{m}^{*}-f_{m}^{* *}\right) u\right\|_{p},
$$

since

$$
f_{m}^{*}=\sum_{k=1}^{j} \frac{\widetilde{l_{i}}}{\mu_{i}} a_{i}^{*}
$$

To estimate the first addendum we use (3.3). For the second one, taking into account (2.6) and (5.9), we can write

$$
\begin{aligned}
\left\|\left(f_{m}^{*}-f_{m}^{* *}\right) u\right\|_{p} & =\left\|\left[\sum_{k=1}^{j} \tilde{\mu_{k}}\left(a_{k}^{*}-v_{k}^{*}\right)\right] u\right\|_{p} \\
& \leq \mathcal{C}\left(\sum_{k=1}^{j} \Delta x_{k}\left|u\left(x_{k}\right)\right|^{p}\left|\frac{a_{k}^{*}-v_{k}^{*}}{\mu_{k}}\right|^{p}\right)^{1 / p} \\
& =\mathcal{C}\left\|\mathbf{a}^{*}-\mathbf{v}^{*}\right\|_{p} \leq \mathcal{C}\left(\frac{\sqrt{a_{m}}}{m}\right)^{r}\left\|\mathbf{a}^{*}\right\|_{l_{p}} .
\end{aligned}
$$

On the other hand, by (5.2), we have

$$
\begin{aligned}
\left\|\mathbf{a}^{*}\right\|_{l_{p}} & =\left(\sum_{k=1}^{j} \Delta x_{k}\left|f_{m}^{*}\left(x_{k}\right) u\left(x_{k}\right)\right|^{p}\right)^{1 / p} \\
& \leq \mathcal{C}\left(\int_{x_{1}}^{\theta_{1} a_{m}}\left|f_{m}^{*}(x) u(x)\right|^{p} d x\right) \\
& \leq \mathcal{C}\left\|f_{m}^{*} u\right\|_{p} \\
& \leq \mathcal{C}\left[\left\|\left(f^{*}-f_{m}^{*}\right) u\right\|_{p}+\left\|f^{*} u\right\|_{p}\right] \\
& \leq \mathcal{C}\left\|f^{*} u\right\|_{p}
\end{aligned}
$$

for sufficiently large $m$. Therefore,

$$
\left\|\left(f_{m}^{*}-f_{m}^{* *}\right) u\right\|_{p} \leq \mathcal{C}\left(\frac{\sqrt{a_{m}}}{m}\right)^{r}\|g\|_{W_{r}^{p}(u)} .
$$

The following proposition completes the proof of the theorem. 
Proposition 5.3. Under the assumptions of Theorem 3.3 we have

$$
\left\|E_{j}\right\| \leq \mathcal{C}\left(\frac{\sqrt{a_{m}}}{m}\right)^{r},
$$

where the matrix norm is that induced by the vector $l_{p}$-norm and the constant $\mathcal{C}$ is independent of $m$.

To prove Proposition 5.3 we use the following result.

Let $L_{m}(w, F)$ denote the Lagrange polynomial interpolating $F$ on the zeros $x_{1}, x_{2}, \ldots, x_{m}$.

Lemma 5.4. Let $1<q<+\infty$ and $\theta \in(0,1)$. If the weights $w(x)=x^{\alpha} e^{-x^{\beta}}$ and $u(x)=x^{\gamma} e^{-x^{\beta} / 2}$ satisfy the conditions (2.13), then for $f \in L_{w / u}^{\infty}$ one has

$$
\left\|L_{m}(w, f) \frac{w}{u}\right\|_{L^{q}\left(\left[0, \theta a_{m}\right)\right)} \leq \mathcal{C}\left(a_{m} m^{2}\right)^{1 / q} \log m\left\|f \frac{w}{u}\right\|_{\infty}
$$

with $C$ independent of $f$ and $m$.

Proof. By applying a Remez-type inequality (see $[\mathbf{1 4},(2.5)]$ ) we can write

$$
\begin{aligned}
\left\|L_{m}(w, f) \frac{w}{u}\right\|_{L^{q}\left(\left[0, \theta a_{m}\right)\right)} & \leq \mathcal{C}\left(\int_{x_{1}}^{a_{m}}\left|L_{m}(w, f ; x) \frac{w(x)}{u(x)}\right|^{q}\right)^{1 / q} \\
& \leq a_{m}^{1 / q}\left\|f \frac{w}{u}\right\|_{\infty} \sup _{x \in\left[x_{1}, a_{m}\right]} \sum_{k=1}^{m}\left|l_{k}(x) \frac{w(x)}{u(x)} \frac{u\left(x_{k}\right)}{w\left(x_{k}\right)}\right| .
\end{aligned}
$$

Then we go to estimate

$$
\sum_{k=1}^{m}\left|l_{k}(x) \frac{w(x)}{u(x)} \frac{u\left(x_{k}\right)}{w\left(x_{k}\right)}\right|=l_{d}(x) \frac{w(x)}{u(x)} \frac{u\left(x_{d}\right)}{w\left(x_{d}\right)}+\sum_{\substack{k=1 \\ k \neq d}}^{m}\left|l_{k}(x) \frac{w(x)}{u(x)} \frac{u\left(x_{k}\right)}{w\left(x_{k}\right)}\right|
$$

where $x_{d}, d \in\{1, \ldots, m\}$ denotes the knot closest to $x$. Since

$$
\frac{l_{d}(x) \sqrt{w(x)}}{\sqrt{w\left(x_{d}\right)}} \sim 1
$$


(see $[8])$ implies

$$
l_{d}(x) \frac{w(x)}{u(x)} \frac{u\left(x_{d}\right)}{w\left(x_{d}\right)} \sim 1
$$

and since the following inequalities hold (see $[8]$ )

$$
\begin{gathered}
\frac{1}{\left|p_{m}^{\prime}\left(w, x_{k}\right) \sqrt{w\left(x_{k}\right)}\right|} \sim \sqrt[4]{a_{m} x_{k}} \Delta x_{k} \sqrt{1-\frac{x_{k}}{a_{m}}+\frac{1}{m^{2 / 3}}} \\
k=1, \ldots, m
\end{gathered}
$$

with constants involved in $\sim$ independent of $m$ and $k, \Delta x_{k}=x_{k+1}-x_{k}$ and

$$
\left|p_{m}(w, x) \sqrt{w(x)}\right| \leq \frac{\mathcal{C}}{\sqrt[4]{a_{m} x} \sqrt[4]{\left|1-\left(x / a_{m}\right)\right|+\left(1 / m^{2 / 3}\right)}}
$$

with $\mathcal{C}\left(a_{m} / m^{2}\right) \leq x \leq \mathcal{C} a_{m}\left(1+m^{-2 / 3}\right), \mathcal{C} \neq \mathcal{C}(m, x)$, we can write, for $x \in\left[x_{1}, a_{m}\right]$,

$$
\begin{aligned}
& \sum_{k=1}^{m}\left|l_{k}(x) \frac{w(x)}{u(x)} \frac{u\left(x_{k}\right)}{w\left(x_{k}\right)}\right| \\
\leq & \mathcal{C}\left(1+x^{(\alpha / 2)-(1 / 4)-\gamma} \sum_{k=1}^{m} \frac{\Delta x_{k} \sqrt{1-\left(x_{k} / a_{m}\right)+\left(1 / m^{2 / 3}\right)}}{x_{k}^{(\alpha / 2)-(1 / 4)-\gamma}\left|x-x_{k}\right|}\right) \\
\leq & \mathcal{C}\left(1+\left(\frac{x_{m}}{x}\right)^{1 / q} x^{(\alpha / 2)-(1 / 4)-\gamma+(1 / q)} \sum_{k=1}^{m} \frac{\Delta x_{k}}{x_{k}^{(\alpha / 2)-(1 / 4)-\gamma+(1 / q)}\left|x-x_{k}\right|}\right) \\
\leq & \mathcal{C}\left(\frac{a_{m}}{a_{m} / m^{2}}\right)^{1 / q} \log m
\end{aligned}
$$

(see, also, [11]) from which the assertion follows.

Proof of Proposition 5.3. By definition, applying Hölder's inequality, we have

$$
\left\|E_{j}\right\|=\sup _{\|\mathbf{a}\|_{l_{p}=1}}\left\|E_{j} \mathbf{a}\right\|_{l_{p}} \leq\left(\left.\left.\sum_{i=1}^{j}\left|\sum_{k=1}^{j}\right| \varepsilon_{i k}\right|^{q}\right|^{p / q}\right)^{1 / p}
$$


where $(1 / p)+(1 / q)=1$ and $\varepsilon_{i k}$ as in (5.7), from which we can deduce

$$
\begin{aligned}
\left\|E_{j}\right\| & \leq\left(\sum _ { i = 1 } ^ { j } \mu _ { i } ^ { p } | \sum _ { k = 1 } ^ { j } | \frac { \lambda _ { k } ( w ) } { \mu _ { k } ( a _ { m } - x _ { k } ) } \left[S_{m}\left(w,\left(k_{x_{i}}\right)_{j}\left(a_{m}-\cdot\right) ; x_{k}\right)\right.\right. \\
& \left.\left.\quad-L_{m+1}^{*}\left(w,\left(k_{x_{i}}\right)_{j}\left(a_{m}-\cdot\right) ; x_{k}\right)\right]\left.\left.\right|^{q}\right|^{p / q}\right)^{1 / p} \\
& \frac{\mathcal{C}}{a_{m}}\left(\sum_{i=1}^{j} \mu_{i}^{p}\left|\sum_{k=1}^{j} \Delta x_{k}\left[\frac{w\left(x_{k}\right)}{u\left(x_{k}\right)}\right]^{q}\right| S_{m}\left(w,\left(k_{x_{i}}\right)_{j}\left(a_{m}-\cdot\right) ; x_{k}\right)\right. \\
= & \frac{\mathcal{C}}{a_{m}}\left(\sum_{i=1}^{j} \mu_{i}^{p} A_{i}^{p}\right)^{1 / p}
\end{aligned}
$$

having used the following relations: $x_{k} \leq x_{j} \leq \bar{\theta} a_{m}$, for a suitable $\bar{\theta} \in(0,1)$ and $\lambda_{k}(w) \sim \Delta x_{k} w\left(x_{k}\right)$ (see [9]). Then let us estimate $A_{i}$ for a fixed $i \in\{1, \ldots, j\}$.

To this end, we define the integer $M=\left[(\theta / \theta+1)^{\beta} m\right] \sim m$ and introduce the polynomial $Q(x)=P_{M-1}(x)\left(a_{m}-x\right)$ with $P_{M-1} \in \mathbb{P}_{M-1}$ the best approximation polynomial of the function $k_{x_{i}}$ in $L_{w / u}^{q}$. We can write

$$
\begin{aligned}
A_{i}= & \left(\sum_{k=1}^{j} \Delta x_{k}\left[\frac{w\left(x_{k}\right)}{u\left(x_{k}\right)}\right]^{q} \mid S_{m}\left(w,\left(k_{x_{i}}\right)_{j}\left(a_{m}-\cdot\right)-Q ; x_{k}\right)\right. \\
\left.-\left.L_{m+1}^{*}\left(w,\left(k_{x_{i}}\right)_{j}\left(a_{m}-\cdot\right)-Q ; x_{k}\right)\right|^{q}\right)^{1 / q} & \\
\leq & \left(\sum_{k=1}^{j} \Delta x_{k}\left[\frac{w\left(x_{k}\right)}{u\left(x_{k}\right)}\right]^{q}\left|S_{m}\left(w,\left(k_{x_{i}}\right)_{j}\left(a_{m}-\cdot\right)-Q ; x_{k}\right)\right|^{q}\right)^{1 / q} \\
& +\left(\sum_{k=1}^{j} \Delta x_{k}\left[\frac{w\left(x_{k}\right)}{u\left(x_{k}\right)}\right]^{q}\left|L_{m+1}^{*}\left(w,\left(k_{x_{i}}\right)_{j}\left(a_{m}-\cdot\right)-Q ; x_{k}\right)\right|^{q}\right)^{1 / q} \\
\leq & \left\|S_{m}\left(w,\left(k_{x_{i}}\right)_{j}\left(a_{m}-\cdot\right)-Q\right) \frac{w}{u}\right\|_{L^{q}\left(\left(0, \theta_{1} a_{m}\right)\right)} \\
& +\left\|L_{m+1}^{*}\left(w,\left(k_{x_{i}}\right)_{j}\left(a_{m}-\cdot\right)-Q\right) \frac{w}{u}\right\|_{L^{q}\left(\left(0, \theta_{1} a_{m}\right)\right)}=: A_{i, 1}+A_{i, 2}
\end{aligned}
$$


having used inequality (5.2). Now, since

$$
\begin{aligned}
\left(k_{x_{i}}\right)_{j}(x)\left(a_{m}-x\right)-Q & (x) \\
= & \left(k_{x_{i}}\right)_{j}(x)\left(a_{m}-x\right)-\left(P_{M-1}\right)_{j}(x)\left(a_{m}-x\right) \\
& +\left(1-\Phi_{j}(x)\right) P_{M-1}(x)\left(a_{m}-x\right)
\end{aligned}
$$

with $\Phi_{j}$ the characteristic function of the interval $\left[0, x_{j}\right]$, we get

$$
\begin{aligned}
A_{i, 1} \leq & \left\|S_{m}\left(w,\left(k_{x_{i}}-P_{M-1}\right)_{j}\left(a_{m}-\cdot\right)\right) \frac{w}{u}\right\|_{L^{q}\left(\left(0, \theta_{1} a_{m}\right)\right)} \\
& +\left\|S_{m}\left(w,\left(1-\Phi_{j}\right) P_{M-1}\left(a_{m}-\cdot\right)\right) \frac{w}{u}\right\|_{L^{q}\left(\left(0, \theta_{1} a_{m}\right)\right)}
\end{aligned}
$$

and, analogously,

$$
\begin{aligned}
A_{i, 2} \leq & \left\|L_{m+1}^{*}\left(w,\left(k_{x_{i}}-P_{M-1}\right)_{j}\left(a_{m}-\cdot\right)\right) \frac{w}{u}\right\|_{L^{q}\left(\left(0, \theta_{1} a_{m}\right)\right)} \\
& +\left\|L_{m+1}^{*}\left(w,\left(1-\Phi_{j}\right) P_{M-1}\left(a_{m}-\cdot\right)\right) \frac{w}{u}\right\|_{L^{q}\left(\left(0, \theta_{1} a_{m}\right)\right)}
\end{aligned}
$$

By applying estimate (2.12) we deduce

$$
\begin{aligned}
\left\|S_{m}\left(w,\left(k_{x_{i}}-P_{M-1}\right)_{j}\left(a_{m}-\cdot\right)\right) \frac{w}{u}\right\|_{L^{q}\left(\left(0, \theta_{1} a_{m}\right)\right)} \\
\leq \mathcal{C}\left\|\left(k_{x_{i}}-P_{M-1}\right)\left(a_{m}-\cdot\right) \frac{w}{u}\right\|_{L^{q}\left(\left(0, x_{j}\right)\right)} \\
\leq \mathcal{C} a_{m}\left\|\left(k_{x_{i}}-P_{M-1}\right) \frac{w}{u}\right\|_{q} \\
=\mathcal{C} a_{m} E_{M-1}\left(k_{x_{i}}\right)_{(w / u), q} \\
\leq \mathcal{C} a_{m}\left(\frac{\sqrt{a_{m}}}{m}\right)^{r}\left\|k_{x_{i}}\right\|_{W_{r}^{q}(w / u)}
\end{aligned}
$$

while, by applying (2.11) and the following inequality ([14])

$$
\begin{aligned}
\left(\int_{(1+\delta) a_{m}}^{\infty}\left|P_{m}(x) u(x)\right|^{p}\right. & d x)^{1 / p} \\
& \leq \mathcal{C} e^{-A m}\left(\int_{0}^{\infty}\left|P_{m}(x) u(x)\right|^{p} d x\right)^{1 / p}
\end{aligned}
$$


that holds for all polynomials $P_{m}$, with $A, \mathcal{C}$ positive constants independent of $m$ and $p$ and $A$ depending on $\delta>0$, we can deduce

$$
\begin{aligned}
\| S_{m}\left(w,\left(1-\Phi_{j}\right)\right. & \left.P_{M-1}\left(a_{m}-\cdot\right)\right) \frac{w}{u} \|_{L^{q}\left(\left(0, \theta_{1} a_{m}\right)\right)} \\
& \leq\left\|S_{m}\left(w,\left(1-\Phi_{j}\right) P_{M-1}\left(a_{m}-\cdot\right)\right) \frac{w}{u}\right\|_{q} \\
& \leq \mathcal{C} m^{1 / 3}\left\|\left(1-\Phi_{j}\right) P_{M-1}\left(a_{m}-\cdot\right) \frac{w}{u}\right\|_{q} \\
& =\mathcal{C} m^{1 / 3}\left(\int_{x_{j}}^{\infty}\left|P_{M-1}(x)\left(a_{m}-x\right) \frac{w(x)}{u(x)}\right|^{q} d x\right)^{1 / q} \\
& \leq \mathcal{C} m^{1 / 3} e^{-A m}\left\|P_{M-1}\left(a_{m}-\cdot\right) \frac{w}{u}\right\|_{q} \cdot
\end{aligned}
$$

Now since, for an arbitrary polynomial $P_{m}$ it also holds $[\mathbf{1 4}]$

$$
\left(\int_{0}^{\infty}\left|P_{m}(x) u(x)\right|^{p} d x\right)^{1 / p} \leq \mathcal{C}\left(\int_{0}^{a_{m}}\left|P_{m}(x) u(x)\right|^{p} d x\right)^{1 / p}
$$

we obtain

$$
\begin{aligned}
\| S_{m}\left(w,\left(1-\Phi_{j}\right)\right. & \left.P_{M-1}\left(a_{m}-\cdot\right)\right) \frac{w}{u} \|_{L^{q}\left(\left(0, \theta_{1} a_{m}\right)\right)} \\
& \leq \mathcal{C} m^{1 / 3} e^{-A m}\left\|P_{M-1}\left(a_{m}-\cdot\right) \frac{w}{u}\right\|_{L^{q}\left(\left(0, a_{m}\right)\right)} \\
& \leq \mathcal{C} a_{m} m^{1 / 3} e^{-A m}\left\|P_{M-1} \frac{w}{u}\right\|_{q} \\
& \leq \mathcal{C} a_{m} m^{1 / 3} e^{-A m}\left\|k_{x_{i}} \frac{w}{u}\right\|_{q} .
\end{aligned}
$$

Then we can conclude

$$
A_{i, 1} \leq \mathcal{C} a_{m}\left(\frac{\sqrt{a_{m}}}{m}\right)^{r}\left\|k_{x_{i}}\right\|_{W_{r}^{q}(w / u)}
$$

Now we want to estimate $A_{i, 2}$. We can write

$$
\begin{aligned}
L_{m+1}^{*}\left(w,\left(k_{x_{i}}\right)_{j}\left(a_{m}\right.\right. & \left.-\cdot)-\left(P_{M-1}\right)_{j}\left(a_{m}-\cdot\right) ; x\right) \\
& =\sum_{k=1}^{j}\left[k_{x_{i}}\left(x_{k}\right)-P_{M-1}\left(x_{k}\right)\right]\left(a_{m}-x_{k}\right) \widetilde{l_{k}}(x) \\
& =\left(a_{m}-x\right) \sum_{k=1}^{j}\left[k_{x_{i}}\left(x_{k}\right)-P_{M-1}\left(x_{k}\right)\right] l_{k}(x)
\end{aligned}
$$


and, then,

$$
\begin{aligned}
& \left\|L_{m+1}^{*}\left(w,\left(k_{x_{i}}\right)_{j}\left(a_{m}-\cdot\right)-\left(P_{M-1}\right)_{j}\left(a_{m}-\cdot\right)\right) \frac{w}{u}\right\|_{L^{q}\left(\left(0, \theta_{1} a_{m}\right)\right)} \\
& \quad=\left(\int_{0}^{\theta_{1} a_{m}}\left(a_{m}-x\right)^{q}\left|\sum_{k=1}^{j}\left[k_{x_{i}}\left(x_{k}\right)-P_{M-1}\left(x_{k}\right)\right] l_{k}(x) \frac{w(x)}{u(x)}\right|^{q} d x\right)^{1 / q} \\
& \quad \leq a_{m}\left(\int_{0}^{\theta_{1} a_{m}}\left|\sum_{k=1}^{j}\left[k_{x_{i}}\left(x_{k}\right)-P_{M-1}\left(x_{k}\right)\right] l_{k}(x) \frac{w(x)}{u(x)}\right|^{q} d x\right)^{1 / q} \\
& \quad=a_{m}\left\|L_{m}\left(w,\left(k_{x_{i}}-P_{M-1}\right)_{j}\right) \frac{w}{u}\right\|_{q} .
\end{aligned}
$$

where we denoted by $L_{m}(w, F)$ the Lagrange polynomial interpolating $F$ on the nodes $x_{1}, x_{2}, \ldots, x_{m}$.

By using estimates proved in [14] (see Theorem 5.6 and Lemma 5.7) and by proceeding as in [7] (see the proof of Theorem 4.2), it follows

$$
\begin{aligned}
& \left\|L_{m+1}^{*}\left(w,\left(k_{x_{i}}-P_{M-1}\right)_{j}\left(a_{m}-\cdot\right)\right) \frac{w}{u}\right\|_{L^{q}\left(\left(0, \theta_{1} a_{m}\right)\right)} \\
& \leq \mathcal{C} a_{m}\left[E_{M-1}\left(k_{x_{i}}\right)_{(w / u), q}+\left(\frac{\sqrt{a_{m}}}{m}\right)^{1 / q} \int_{0}^{\sqrt{a_{m}} / m} \frac{\Omega_{\varphi}^{r}\left(k_{x_{i}}, t\right)_{(w / u), q}}{t^{1+(1 / q)}} d t\right] \\
& \leq \mathcal{C} a_{m}\left[E_{M-1}\left(k_{x_{i}}\right)_{(w / u), q}+\left(\frac{\sqrt{a_{m}}}{m}\right)^{r}\left\|k_{x_{i}}^{(r)} \varphi^{r} \frac{w}{u}\right\|_{q}\right] \\
& \leq \mathcal{C} a_{m}\left(\frac{\sqrt{a_{m}}}{m}\right)^{r}\left\|k_{x_{i}}\right\|_{W_{q}^{r}(w / u)} .
\end{aligned}
$$

It remains to estimate the second addendum of $A_{i, 2}$. We have

$$
\begin{aligned}
L_{m+1}^{*}\left(w,\left(1-\Phi_{j}\right) P_{M-1}\left(a_{m}-\cdot\right) ; x\right) & \\
& =\sum_{k=j+1}^{m+1} P_{M-1}\left(x_{k}\right)\left(a_{m}-x_{k}\right) \tilde{l_{k}}(x) \\
& =\left(a_{m}-x\right) \sum_{k=j+1}^{m+1} P_{M-1}\left(x_{k}\right) l_{k}(x) .
\end{aligned}
$$


Then one has

$$
\begin{aligned}
\| L_{m+1}^{*}( & \left(w,\left(1-\Phi_{j}\right) P_{M-1}\left(a_{m}-\cdot\right)\right) \frac{w}{u} \|_{L^{q}\left(\left(0, \theta_{1} a_{m}\right)\right)} \\
= & \left(\int_{0}^{\theta_{1} a_{m}}\left(a_{m}-x\right)^{q}\left|\sum_{k=j+1}^{m+1} P_{M-1}\left(x_{k}\right) l_{k}(x) \frac{w(x)}{u(x)} d x\right|^{q}\right)^{1 / q} \\
& \leq a_{m}\left(\int_{0}^{\theta_{1} a_{m}}\left|\sum_{k=j+1}^{m+1} P_{M-1}\left(x_{k}\right) l_{k}(x) \frac{w(x)}{u(x)} d x\right|^{q}\right)^{1 / q} \\
& =a_{m}\left\|L_{m}\left(w,\left(1-\Phi_{j}\right) P_{M-1}\left(a_{m}-\cdot\right)\right) \frac{w}{u}\right\|_{L^{q}\left(\left(0, \theta_{1} a_{m}\right)\right)}
\end{aligned}
$$

By using Lemma 5.4, estimate (5.1) and also, by applying the Nikolski inequality (see [14])

$$
\left\|Q_{m} \frac{w}{u}\right\|_{\infty} \leq \mathcal{C}\left(\frac{m}{\sqrt{a_{m}}}\right)^{2 / q}\left\|Q_{m} \frac{w}{u}\right\|_{q},
$$

holding for $Q_{m} \in \mathbb{P}_{m}$ and $\mathcal{C} \neq \mathcal{C}\left(m, q, Q_{m}\right)$, we can write

$$
\begin{aligned}
\| L_{m+1}^{*}(w, & \left.\left(1-\Phi_{j}\right) P_{M-1}\left(a_{m}-\cdot\right)\right) \frac{w}{u} \|_{L^{q}\left(\left(0, \theta_{1} a_{m}\right)\right)} \\
& \leq \mathcal{C} a_{m}\left(a_{m} m^{2}\right)^{1 / q}(\log m)\left\|\left(1-\Phi_{j}\right) P_{M-1} \frac{w}{u}\right\|_{\infty} \\
& \leq \mathcal{C} a_{m}\left(a_{m} m^{2}\right)^{1 / q}(\log m) e^{-A m}\left\|P_{M-1} \frac{w}{u}\right\|_{\infty} \\
& \leq \mathcal{C} a_{m}\left(a_{m} m^{2}\right)^{1 / q}(\log m) e^{-A m}\left(\frac{m}{\sqrt{a_{m}}}\right)^{2 / q}\left\|P_{M-1} \frac{w}{u}\right\|_{q} \\
& \leq \mathcal{C} a_{m} m^{4 / q}(\log m) e^{-A m}\left\|k_{x_{i}} \frac{w}{u}\right\|_{q} .
\end{aligned}
$$

From the previous results we can deduce

$$
A_{i, 2} \leq \mathcal{C} a_{m}\left(\frac{\sqrt{a_{m}}}{m}\right)^{r}\left\|k_{x_{i}}\right\|_{W_{r}^{q}(w / u)}
$$

and combining (5.15) and (5.16)

$$
A_{i} \leq \mathcal{C} a_{m}\left(\frac{\sqrt{a_{m}}}{m}\right)^{r}\left\|k_{x_{i}}\right\|_{W_{r}^{q}(w / u)} .
$$


Therefore, since for $F \in W_{r}^{p}(u)$ (see [14])

$$
\Omega_{\varphi}^{r}(F, t)_{u, p} \leq \mathcal{C} t^{r}\left\|F^{(r)} \varphi^{r} u\right\|_{p}, \quad \varphi(x)=\sqrt{x}
$$

by using (2.8), we can obtain

$$
\begin{aligned}
\left\|E_{j}\right\| \leq & \frac{\mathcal{C}}{a_{m}}\left(\sum_{i=1}^{j} \mu_{i}^{p} A_{i}^{p}\right)^{1 / p} \\
\leq & \mathcal{C}\left(\frac{\sqrt{a_{m}}}{m}\right)^{r}\left(\sum_{i=1}^{j} \Delta x_{i}\left|u\left(x_{i}\right)\right|^{p}\left\|k_{x_{i}}\right\|_{W_{r}^{q}(w / u)}^{p}\right)^{1 / p} \\
\leq & \mathcal{C}\left(\frac{\sqrt{a_{m}}}{m}\right)^{r}\left[\left(\int_{0}^{\infty}\left|\left\|k_{y}\right\|_{W_{r}^{q}(w / u)} u(y)\right|^{p} d y\right)^{1 / p}\right. \\
& \left.+\left(\frac{\sqrt{a_{m}}}{m}\right)^{r}\left(\int_{0}^{\infty}\left|\frac{\partial^{r}}{\partial y^{r}}\left(\left\|k_{y}\right\|_{W_{r}^{q}(w / u)}\right) \varphi^{r}(y) u(y)\right|^{p} d y\right)^{1 / p}\right] \\
\leq & \mathcal{C}\left(\frac{\sqrt{a_{m}}}{m}\right)^{r},
\end{aligned}
$$

taking into account the assumptions.

Proof of Theorem 3.4. Taking into account relation (4.1.33) in [1], it is sufficient to estimate $\left\|\left[\left(K-\bar{K}_{m}\right) f\right] u\right\|_{p}$. We have

$$
\begin{aligned}
& \left\|\left[\left(K-\bar{K}_{m}\right) f\right] u\right\|_{p} \\
= & \left(\int_{0}^{\infty}\left|u(y) \int_{0}^{\infty}\left[k_{y}(x) f(x)-L_{m+1}^{*}\left(w,\left(k_{y} f\right)_{j} ; x\right)\right] w(x) d x\right|^{p} d y\right)^{1 / p} .
\end{aligned}
$$

Let

$$
M=\left[\left(\frac{\theta}{1+\theta}\right)^{\beta} m\right] \sim m, \quad P_{1} \in \mathbb{P}_{[M / 2]}
$$

be the best approximation polynomial of $f$ in $L_{u}^{p}, P_{2, y} \in \mathbb{P}_{[M / 2]}$ the best approximation polynomial of $k_{y}$ in $L_{w / u}^{q}$, with $1 / p+1 / q=1$, and 


$$
\begin{aligned}
& Q_{y}=P_{1} P_{2, y} \text {. Then, we can write } \\
& \qquad \begin{aligned}
\int_{0}^{\infty}\left[k_{y}(x) f(x)-\right. & \left.L_{m+1}^{*}\left(w,\left(k_{y} f\right)_{j} ; x\right)\right] w(x) d x \\
= & \int_{0}^{\infty}\left[k_{y}(x) f(x)-Q_{y}(x)\right] w(x) d x \\
& +\int_{0}^{\infty}\left[Q_{y}(x)-L_{m+1}^{*}\left(w,\left(k_{y} f\right)_{j} ; x\right)\right] w(x) d x \\
= & : A_{1}(y)+A_{2}(y) .
\end{aligned}
\end{aligned}
$$

In order to estimate $A_{1}(y)$ and $A_{2}(y)$, let us use the following formula

$$
\begin{aligned}
k(x, y) f(x)-Q_{y}(x)= & {\left[f(x) u(x)\left(k(x, y)-P_{2, y}(x)\right)\right.} \\
& \left.+P_{2, y}(x)\left(f(x)-P_{1}(x)\right) u(x)\right] u^{-1}(x) .
\end{aligned}
$$

We obtain, by Hölder's inequality,

$$
\begin{aligned}
\left|A_{1}(y)\right|= & \mid \int_{0}^{\infty} f(x) u(x)\left[k(x, y)-P_{2, y}(x)\right] \frac{w(x)}{u(x)} d x \\
& +\int_{0}^{\infty}\left[f(x)-P_{1}(x)\right] u(x) P_{2, y}(x) \frac{w(x)}{u(x)} d x \mid \\
\leq & \|f u\|_{p}\left(\int_{0}^{\infty}\left|\left[k(x, y)-P_{2, y}(x)\right] \frac{w(x)}{u(x)}\right|^{q} d x\right)^{1 / q} \\
& +\left\|\left(f-P_{1}\right) u\right\|_{p}\left(\int_{0}^{\infty}\left|P_{2, y}(x) \frac{w(x)}{u(x)}\right|^{q} d x\right)^{1 / q} \\
\leq & \mathcal{C}\left(\frac{\sqrt{a_{m}}}{m}\right)^{r}\|f u\|_{W_{r}^{p}(u)}\left\|k_{y}\right\|_{W_{r}^{q}(w / u)},
\end{aligned}
$$

taking into account that, under the assumptions, one has $f \in W_{r}^{p}(u)$, since both $K f \in W_{r}^{p}(u)$ and $g \in W_{r}^{p}(u)$, and estimate (2.10) holds.

Let us estimate, now, $A_{2}(y)$. By applying the Gaussian quadrature rule with respect to the weight function $w(x)$, we have

$$
\begin{aligned}
A_{2}(y) & =\int_{0}^{\infty} L_{m+1}^{*}\left(w, Q_{y}-\left(k_{y} f\right)_{j} ; x\right) w(x) d x \\
& =\sum_{k=1}^{j} \lambda_{k}(w)\left[Q_{y}\left(x_{k}\right)-k_{y}\left(x_{k}\right) f\left(x_{k}\right)\right]+\sum_{k=j+1}^{m} \lambda_{k}(w) Q_{y}\left(x_{k}\right) \\
& =: A_{2,1}(y)+A_{2,2}(y) .
\end{aligned}
$$


By using relation (5.17), with $q$ such that $(1 / p)+(1 / q)=1$, we get for $A_{2,1}(y)$

$$
\begin{aligned}
A_{2,1}(y) & =\sum_{k=1}^{j} \frac{\lambda_{k}^{1 / p}(w)}{w^{1 / p}\left(x_{k}\right)} f\left(x_{k}\right) u\left(x_{k}\right) \frac{\lambda_{k}^{1 / q}(w)}{w^{1 / q}\left(x_{k}\right)}\left[P_{2, y}\left(x_{k}\right)-k\left(x_{k}, y\right)\right] \frac{w\left(x_{k}\right)}{u\left(x_{k}\right)} \\
& +\sum_{k=1}^{j} \frac{\lambda_{k}^{1 / p}(w)}{w^{1 / p}\left(x_{k}\right)}\left[P_{1}\left(x_{k}\right)-f\left(x_{k}\right)\right] u\left(x_{k}\right) \frac{\lambda_{k}^{1 / q}(w)}{w^{1 / q}\left(x_{k}\right)} P_{2, y}\left(x_{k}\right) \frac{w\left(x_{k}\right)}{u\left(x_{k}\right)} .
\end{aligned}
$$

Now, if we apply Hölder's inequality and use equivalence (3.12), we get

$$
\begin{aligned}
\left|A_{2,1}(y)\right| \leq & \left(\sum_{k=1}^{j} \frac{\lambda_{k}(w)}{w\left(x_{k}\right)}\left|f\left(x_{k}\right) u\left(x_{k}\right)\right|^{p}\right)^{1 / p} \\
& +\left(\sum_{k=1}^{j} \frac{\lambda_{k}(w)}{w\left(x_{k}\right)}\left|\left[P_{2, y}\left(x_{k}\right)-k\left(x_{k}, y\right)\right] \frac{w\left(x_{k}\right)}{u\left(x_{k}\right)}\right|^{q}\right)^{1 / q} \\
\leq & \left.\mathcal{C}\left(\left.\sum_{k=1}^{j} \Delta x_{k}\left|f\left(x_{k}\right)\right|\left[P_{1}\left(x_{k}\right)-f\left(x_{k}\right)\right] u\left(x_{k}\right)\right|^{p}\right)^{1 / p} \frac{\lambda_{k}(w)}{w\left(x_{k}\right)}\left|P_{2, y}\left(x_{k}\right) \frac{w\left(x_{k}\right)}{u\left(x_{k}\right)}\right|^{q}\right)^{1 / q} \\
& +\left(\sum_{k=1}^{j} \Delta x_{k}\left|\left[P_{2, y}\left(x_{k}\right)-k\left(x_{k}, y\right)\right] \frac{w\left(x_{k}\right)}{u\left(x_{k}\right)}\right|^{q}\right)^{1 / q} \\
& \cdot\left(\sum_{k=1}^{j} \Delta x_{k}\left|\left[P_{1}\left(x_{k}\right)-f\left(x_{k}\right)\right] u\left(x_{k}\right)\right|^{p}\right)^{1 / p} \\
& \\
& \\
& \\
&
\end{aligned}
$$

From (5.2), (2.2) and (2.10) it follows

$$
\left|A_{2,1}(y)\right| \leq \mathcal{C}\left(\frac{\sqrt{a_{m}}}{m}\right)^{r}\|f u\|_{W_{r}^{p}(u)}\left\|k_{y}\right\|_{W_{r}^{q}(w / u)} .
$$


It remains to estimate $A_{2,2}(y)$. To this end we recall that ([14])

$$
\Delta x_{k} \sim \frac{\sqrt{a_{m}}}{m} \sqrt{x_{k}} \sqrt{\frac{a_{m}}{a_{m}-x_{k}+\left(a_{m} / m^{2 / 3}\right)}}, \quad k=1,2, \ldots, m,
$$

from which we get

$$
\Delta x_{k} \leq \mathcal{C} \frac{\sqrt{a_{m}}}{m} \sqrt{x_{k}} m^{1 / 3}, \quad k \geq j+1 .
$$

Set $\tau_{m, k}=\mathcal{C}\left(\sqrt{a_{m}} / m\right) \sqrt{x_{k}}$ with the constant $\mathcal{C}$ such that $x_{k}+\tau_{m, k}<$ $x_{k+1}$, and it also holds (see [13]) that $w(x) \sim w(t)$ and $\sqrt{x} \sim \sqrt{t}$ for $x, t \in\left[x_{k}, x_{k}+\tau_{m, k}\right]$. Then, from the identity

$$
\tau_{m, k} Q_{y}\left(x_{k}\right)=\int_{x_{k}}^{x_{k}+\tau_{m, k}} Q_{y}(t) d t-\int_{x_{k}}^{x_{k}+\tau_{m, k}}\left(x_{k}+\tau_{m, k}-t\right) Q_{y}^{\prime}(t) d t
$$

we get, for $k>j$,

$$
\begin{aligned}
& \lambda_{k}(w)\left|Q_{y}\left(x_{k}\right)\right| \leq \mathcal{C} m^{1 / 3} \tau_{m, k}\left|Q_{y}\left(x_{k}\right) w\left(x_{k}\right)\right| \\
& \leq \mathcal{C} m^{1 / 3}\left(\int_{x_{k}}^{x_{k+1}}\left|Q_{y}(t) w(t)\right| d t+\frac{\sqrt{a_{m}}}{m} \int_{x_{k}}^{x_{k+1}}\left|Q_{y}^{\prime}(t) \sqrt{t} w(t)\right| d t\right) .
\end{aligned}
$$

By summing for $k=j+1, \ldots, m$, one has

$$
\left|A_{2,2}(y)\right| \leq \mathcal{C} m^{1 / 3}\left(\int_{x_{k}}^{\infty}\left|Q_{y}(t) w(t)\right| d t+\frac{\sqrt{a_{m}}}{m} \int_{x_{k}}^{\infty}\left|Q_{y}^{\prime}(t) \sqrt{t} w(t)\right| d t\right) .
$$

Since $Q_{y} \in \mathbb{P}_{M}, M=\left[(\theta / 1+\theta)^{\beta} m\right]$ and $x_{j+1}>\theta a_{m}, \theta \in(0,1)$ fixed, we can apply to both integrals inequality (5.13) to get, with $\varphi(t)=\sqrt{t}$, the estimate

$$
\left|A_{2,2}(y)\right| \leq \mathcal{C} m^{1 / 3} e^{-A m}\left(\left\|Q_{y} w\right\|_{1}+\frac{\sqrt{a_{m}}}{m}\left\|Q_{y}^{\prime} \varphi w\right\|_{1}\right) .
$$

By applying the Bernstein inequality at first, and the Hölder inequality later $([\mathbf{1 3}])$, we get

$$
\begin{aligned}
\left|A_{2,2}(y)\right| & \leq \mathcal{C} m^{1 / 3} e^{-A m}\left\|Q_{y} w\right\|_{1} \\
& \leq 2 \mathcal{C} m^{1 / 3} e^{-A m}\|f u\|_{p}\left\|k_{y} \frac{w}{u}\right\|_{q} \\
& \leq \mathcal{C}\left(\frac{\sqrt{a_{m}}}{m}\right)^{r}\|f u\|_{p}\left\|k_{y} \frac{w}{u}\right\|_{q} \\
& \leq \mathcal{C}\left(\frac{\sqrt{a_{m}}}{m}\right)^{r}\|f\|_{W_{r}^{p}(u)}\left\|k_{y}\right\|_{W_{r}^{q}(w / u)} .
\end{aligned}
$$


Using the estimates proved before for $\left|A_{1}(y)\right|$ and $\left|A_{2}(y)\right|$, and taking into account the hypotheses $(2.22)$ on the kernel $k(x, y)$, we obtain

$$
\begin{aligned}
\|\left[\left(K-\bar{K}_{m}\right) f\right] & u \|_{p} \\
\leq & \left(\int_{0}^{\infty}\left|A_{1}(y) u(y)\right|^{p} d y\right)^{1 / p} \\
& +\left(\int_{0}^{\infty}\left|A_{2}(y) u(y)\right|^{p} d y\right)^{1 / p} \\
\leq & \mathcal{C}\left(\frac{\sqrt{a_{m}}}{m}\right)^{r}\|f\|_{W_{r}^{p}(u)}\left(\int_{0}^{\infty}\left|\left\|k_{y}\right\|_{W_{r}^{q}(w / u)} u(y)\right|^{p} d y\right)^{1 / p} \\
\leq & \mathcal{C}\left(\frac{\sqrt{a_{m}}}{m}\right)^{r}\|f\|_{W_{r}^{p}(u)} .
\end{aligned}
$$

Acknowledgments. The authors wish to thank the referees for their helpful remarks and suggestions.

\section{REFERENCES}

1. K.E. Atkinson, The numerical solution of integral equations of the second kind, Cambridge Mono. Appl. Comput. Math., Cambridge University Press, Cambridge, 1997.

2. A.S. Cvetković and G.V. Milovanović, The mathematica package "OrthogonalPolynomials," Facta Univ. Ser. Math. Inform. 19 (2004), 17-36.

3. M.C. De Bonis and G. Mastroianni, Projection methods and condition numbers in uniform norm for Fredholm and Cauchy singular integral equations, SIAM J. Numer. Anal. 44 (2006), 1351-1374.

4. Z. Ditzian and V. Totik, Moduli of smoothness, SCMG Springer-Verlag, New York, 1987.

5. C. Frammartino, C. Laurita and G. Mastroianni, On the numerical solution of Fredholm integral equations on unbounded intervals, J. Comp. Appl. Math. 158 (2003), 355-378.

6. C. Laurita and G. Mastroianni, Condition numbers in numerical methods for Fredholm integral equations of the second kind, J. Integral Equations Appl. 14 (2002), 311-341.

7. $-L^{p}$ - convergence of Lagrange interpolation on the semiaxis, Acta Math. Hungar. 3 (2008), 249-273.

8. A.L. Levin and D.S. Lubinski, Christoffel Functions, Orthogonal polynomials and Nevai's Conjecture for Freud Weights, Constr. Approx. 8 (1992), 463-535.

9. - Orthogonal Polynomials for weights $x^{2 \rho} e^{-2 Q(x)}$ on $[0, d)$, J. Approx. Theory 134 (2005), 199-256. 
10. G. Mastroianni and G. Monegato, Truncated quadrature rules over $(0, \infty)$ and Nyström-type methods, SIAM J. Numer. Anal. Vol. 41 (2003), 1870-1892.

11. G. Mastroianni and G.V. Milovanović, Interpolation processes: Basic theory and applications, in progress.

12. G. Mastroianni and D. Occorsio, Mean convergence of Fourier sums on unbounded intervals, submitted.

13. G. Mastroianni and J. Szabados, Polynomial approximation on infinite intervals with weights having inner zeros, Acta Math. Hungar. 96 (2002), 221-258.

14. - Polynomial approximation on the real semiaxis with generalized Laguerre weights, submitted.

15. G. Mastroianni and P. Vërtesi, Fourier sums and Lagrange interpolation on $(0,+\infty)$ and $(-\infty,+\infty)$, in Frontiers in Interpolation And Approximation, N.K. Govil, H.N. Mhaskar, R.N. Mohapatra, Z. Nashed and J. Szabados, eds., Taylor \& Francis Books, Boca Raton, Florida, 2006.

16. E.B. Saff and V. Totik, Logarithmic potentials with external fields, Springer, Berlin, (1997).

17. I.M. Sloan, A quadrature-based approach to improving the collocation method, Numer. Math. 54, (1988), 41-56.

18. G. Szegö, Orthogonal polynomials, 4th ed., Amer. Math. Soc. Coll. Publ. 23 American Mathematical Society, Providence, RI, 1975.

Dipartimento di Matematica, Universitá della Basilicata, Via dell'Ateneo Lucano 10, 85100 Potenza, Italy.

Email address: concetta.laurita@unibas.it

Dipartimento di Matematica, Universitá della Basilicata, Via dell'Ateneo Lucano 10, 85100 Potenza, Italy.

Email address: giuseppe.mastroianni@unibas.it 